\title{
Simulations of Turbulent Flows with Strong Shocks and Density Variations
}

\author{
Final Technical Report on
}

DNS of interaction of isotropic turbulence with very strong shocks using high-order shock-fitting method and implementation of non-equilibrium effects

December 2012

Xiaolin Zhong

Mechanical and Aerospace Engineering University of California, Los Angeles, Los Angeles, CA 90095-1597

Phone: (310) 825-2905

e-mail: xiaolin@seas.ucla.edu

Supported by the Department of Energy "Scientific Discovery through Advanced Computing (SciDAC)" program

Program Manager: Dr. Randall Laviolette 
TABLE OF CONTENTS

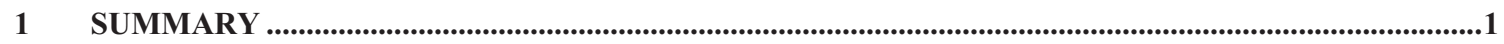

2 INTRODUCTION...............................................................................................................................................2

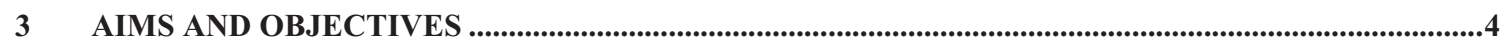

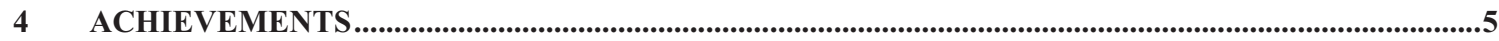

5 IMPLEMENTATION OF SHOCK-FITTING FOR SHOCK-TURBULENCE INTERACTION..............6

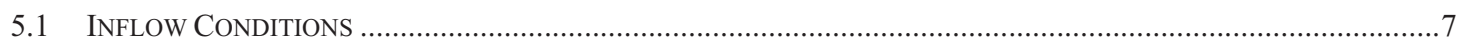

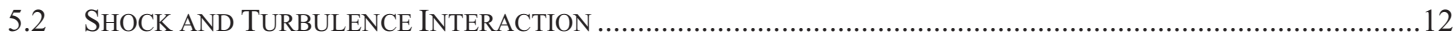

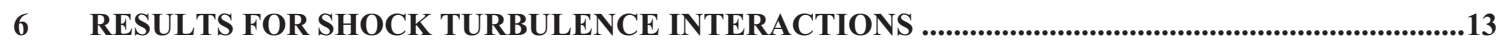

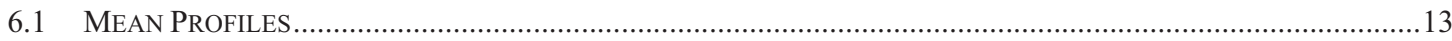

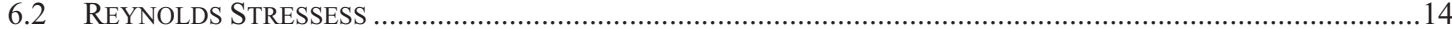

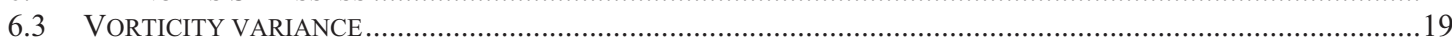

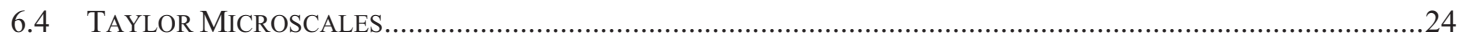

7 DEVELOPMENT AND VALIDATION OF HIGH-ORDER NON-EQUILIBRIUM SHOCK-FITTING

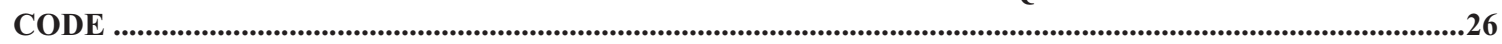

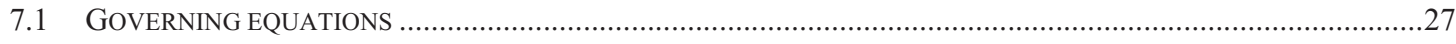

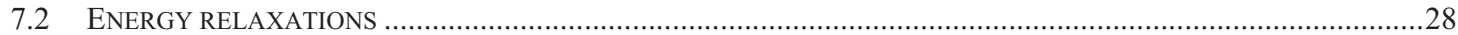

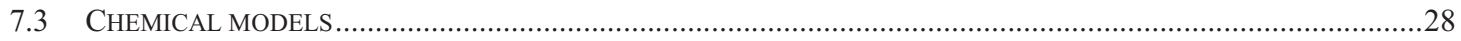

7.4 TRANSPORTATION COEFFICIENTS

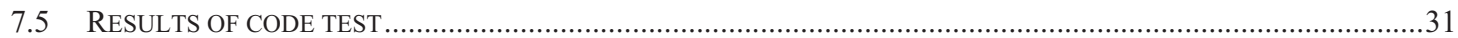

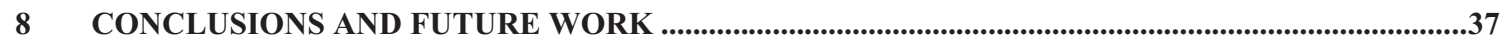

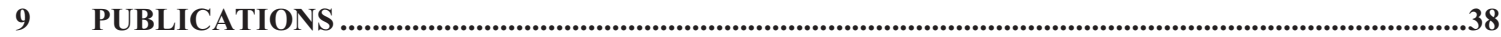

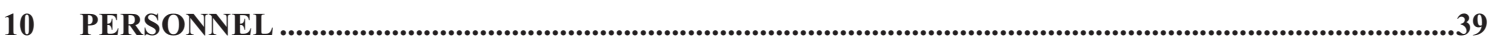

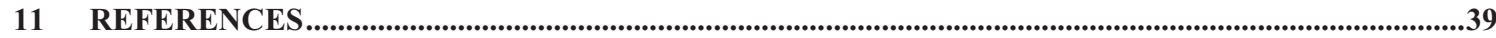




\section{SUMMARY}

In this report, we present the research efforts made by our group at UCLA in the SciDAC project "Simulations of turbulent flows with strong shocks and density variations". We use shock-fitting methodologies as an alternative to shock-capturing schemes for the problems where a well defined shock is present. In past five years, we have focused on development of high-order shock-fitting Navier-Stokes solvers for perfect gas flow and thermochemical non-equilibrium flow and simulation of shock-turbulence interaction physics for very strong shocks. Such simulation has not been possible before because the limitation of conventional shock capturing methods. The limitation of shock Mach number is removed by using our high-order shock-fitting scheme. With the help of DOE and TeraGrid/XSEDE super computing resources, we have obtained new results which show new trends of turbulence statistics behind the shock which were not known before. Moreover, we are also developing tools to consider multi-species nonequilibrium flows. The main results are in three areas: (1) development of high-order shock-fitting scheme for perfect gas flow, (2) Direct Numerical Simulation (DNS) of interaction of realistic turbulence with moderate to very strong shocks using super computing resources, and (3) development and implementation of models for computation of mutli-species non-quilibrium flows with shock-fitting codes.

Based on the goal of the SciDAC team we have been concentrating on the problem of shock and turbulence interaction. We have finished the development and implementation of shock-fitting code for shock and isotropic turbulence interaction for perfect gases. While our SciDAC team members from Stanford University solve these problems using a hybrid scheme, we use shock-fitting scheme which has been shown to be very accurate and stable especially for high Mach number flows. Hence, we complement the efforts of our SciDAC team members by computing flows with moderate to very strong shocks. We applied for and were awarded approximately six million CPU hours at computer resources of DOE-NERSC and TeraGrid/XSEDE. Using these resources, we have computed more than 40 cases by varying different parameters of incoming turbulent flow. Specifically, we have considered flows with mean Mach numbers ranging from 2 to 30 . The turbulent Mach number, $M_{t}$, has been varied from 0.12 to 0.38. Reynolds number based on Taylor microscale, $\operatorname{Re}_{\lambda}$ of upto 52.4 was used which needed more than 50 million grid points. Some new trends were observed in turbulent statistics as we computed high (mean) Mach number flows that have not been computed in past. For example, as shown in following figure, maximum streamwise Reynolds stress values downstream of the shock was generally found to be decreasing as Mach number is increased till about Mach 8. Values match well with those reported by our SciDAC team members at Stanford (Dr. Larsson and Prof. Lele). However, as we computed even stronger shocks this trend reverses and we observe that amplification in streamwise Reynolds stress increases as we increase the shock strength.

For the simulation of turbulent flow interacting with strong shocks, the effects of vibration and electronic energy excitations, translation-vibration energy relaxation, ionization, and chemical reactions among different species need to be considered, because gas temperature increases dramatically after strong shocks. We have been working on the numerical simulations of thermo-chemical non-equilibrium flows in the SAP by implementing state-of-the-art thermo-chemical models of air to general shockcapturing method such as second-order TVD scheme and fifth-order WENO schemes. By shifting from SAP to the Science part, we have implemented to current shock-fitting code the more realistic 11-species model of air with most recent thermo-chemical models of non-equilibrium air flow, including gas-mixture viscosity, heat conductivity, diffusion coefficients, non-equilibrium reaction rates, and equilibrium constants. The non-equilibrium high-order shock-fitting code can run cases with extremely high translational temperature with high accuracy. 


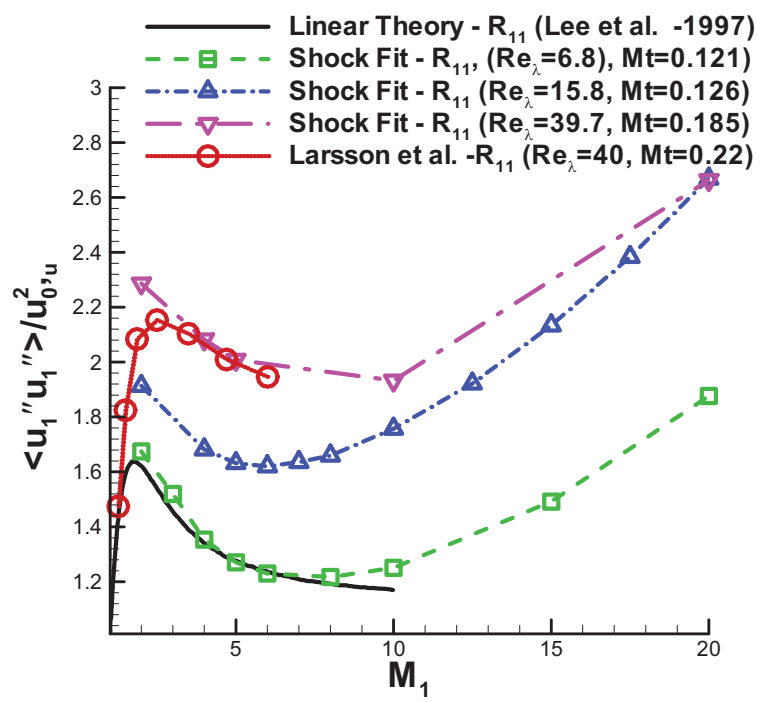

Thus very high Mach number computations using our shock-fitting method provides interesting insight for shock turbulence interaction problem. We have run many cases of the shock turbulence interactions with the perfect gas code to complete the parametric study. This large database of DNS results will be used for understating physics and developing models for shock turbulence interactions. These perfect gas results will also serve as baseline for non-equilibrium results.

\section{INTRODUCTION}

The interactions between turbulent flows and shock waves are important in many natural processes as well as scientific and engineering applications, such as volcanic eruption, supernova explosion, detonation, medical application of shock wave lithotripsy to break up kidney stones, and energy application of the implosion of a cryogenic fuel capsule for inertial confinement fusion where very high rates of compression and expansion waves are generally observed. The underlying physics in shock and turbulence interaction is essential for a better understanding of such processes and applications. Unfortunately, these phenomena are strongly nonlinear and proven to be very complex to understand.

One of the fundamental building blocks in these complex processes and applications is the canonical problem of the interaction of isotropic turbulence and a normal shock, and even this fundamental problem is not well understood for strong shocks. Figure 1 shows a schematic of the canonical strong shock and turbulence interaction problem. In such flows, the coupling between shock wave and turbulent flow is very strong. Complex linear and nonlinear mechanisms are involved which alter the dynamics of the shock motion and can cause considerable changes in the structure of turbulence and its statistical properties. This fundamental shock and turbulence interaction problem has been a challenge for experimentalists, theorists and computational researchers for more than fifty years.

Analytical studies of shock and turbulence interactions have been attempted mostly through linear interaction theory where only small perturbations are considered. Kovasznay [1] showed that, for weak fluctuations of density, pressure, and entropy, turbulent fluctuations about uniform mean flow can be decomposed into acoustic, vorticity, and entropy modes. It was shown that each of these modes evolves independently in the inviscid limit for weak fluctuations. The modifications of random small fluctuations of pressure, entropy and vorticity after passing through shock or flame were studied by Moore [2] and 
Kerrebrock [3]. It was found that acoustic, vorticity, and entropy modes are generated in the downstream flow if any of the modes is presented in the upstream flow. More recent theoretical studies of shock and turbulence interaction were carried out by Goldstein [4], Lee et al. [5, 6], Mahesh et al. [7, 8] and Fabre et al. [9]. It was found in these studies that the RMS values of fluctuating pressure, temperature, and density as well as different components of turbulent kinetic energy are amplified across the shocks. Despite several assumptions, linear interaction theory satisfactorily predicts the essential characteristics of the interaction of shock waves with freestream turbulence/perturbations, and it is only valid for very small perturbations.

Various attempts have been made towards DNS of shock and turbulence interactions since the early 1980s. Initial efforts were focused on the interaction of shock with simple disturbance waves. In 1981, Pao and Salas [10] fitted the shock at inflow boundary and solved Euler equation with finite difference discretization to study a shock/vortex interaction. Shock-fitting computations with pseudospectral (Zang et. al [11]) and spectral techniques (Hussaini et al $[12,13]$ ) were later used to treat the problems in which a single vortex, a vortex sheet, an entropy spot or acoustic wave interacts with the shock. The results obtained from these numerical efforts confirmed the linear theory for weak shocks. With the advent of essentially non-oscillatory (ENO) and related schemes, a number of shock-capturing schemes for compressible flows have been tested for interaction of shock with small disturbances. Although limited to low Mach numbers, these studies mostly confirm the linear interaction analysis results [13-15].

For studies of a fully turbulent flow interacting with shocks, DNS methods and large eddy simulations (LES) have been used. However these different types of methods give different results when interaction with shock is considered [16]. Most of the recent DNS studies have been on various aspects of interaction of a normal shock with freestream turbulence for relatively weak shock at small Mach numbers. For example, Mahesh et al. [7, 8] did extensive DNS studies on the interaction of a normal shock with an isotropic turbulence. The mean shock Mach numbers were in the range of 1.29 to 1.8. They found that the upstream correlation between the vorticity and entropy fluctuations has strong influence on the evolution of the turbulence across the shock. Lee et al. [6] investigated the effect of Mach numbers on isotropic turbulence interacting with a shock wave. The range of Mach numbers was from 1.5 to 3.0. A shock-capturing scheme was developed to simulate the unsteady interaction of turbulence with shock waves. It was found that turbulence kinetic energy is amplified across the shock wave, and this amplification tends to saturate beyond Mach 3. Hannapel et al. [17] computed shock and turbulence interaction of a Mach 2 shock with a third-order shock-capturing scheme based on the essentially nonoscillatory (ENO) algorithm. Jamme et al. [18] carried out a DNS study of the interaction between normal shock waves of moderate strength (Mach 1.2 and Mach 1.5) and isotropic turbulence. Adams and Shariff $[19,20]$ proposed a class of upwind-biased finite-difference schemes with a compact stencil for shock and turbulence interaction simulation. They used the non-conservative upwind scheme in smooth region while a shock-capturing ENO scheme was turned on around discontinuities. This idea of hybrid formulation was improved by Pirozzoli [21] who used similar hybrid formulation for a compact weighted essentially non-oscillatory (WENO) scheme with conservative formulation for simulation of shock and turbulence interaction. Ducros et al. [22] conducted LES studies on shock and turbulence interaction by using a second-order finite volume scheme. The method was then used to simulate the interaction of a Mach 1.2 shock with homogeneous turbulence.

It is noticed that flows with stronger than Mach 3 shocks have not been considered in the past for shock and turbulence interaction problems. High-order shock-capturing schemes have been the methods of choice in most previous numerical simulation studies [7, 8, 23, 24]. However, popular shock-capturing schemes are not very accurate in this regard as they inherently use numerical dissipation in the whole computational domain. Moreover, spurious numerical oscillations have been observed when solving strong shock and turbulence interaction problems with shock-capturing schemes [25]. In shock-capturing 
schemes, the shock generally spreads over a few grid points. With strong shocks, the thickness of the shock front decreases which requires more resolution for shock-capturing schemes. Thus, constraint due to choice of algorithms has been one of the main limitations in past studies. DNS results are currently available for $\operatorname{Re}_{\lambda}=12-22$, where $\operatorname{Re}_{\lambda}$ is Reynolds number based Taylor microscale $\lambda$. However, the typical Reynolds number in real shock and turbulence interaction experiments are $\operatorname{Re}_{\lambda}=200-750$ [26]. The highest Reynolds number of flow that can be resolved using DNS is bounded by the available computational resources. It was estimated that for DNS of shock and turbulence interaction with $\operatorname{Re}_{\lambda} \approx 100$ around $19 \times 10^{9}$ grid points were needed [27]. Prohibitively large computational resources are needed for better understanding of realistic flow situations and inadequate computational resources have been another limitation in past studies.

Literature review of shock and turbulence interactions shows that these complex configurations are part of a number of important applications but the current scientific understanding of strong shock and turbulence interactions in complex configurations and the ability to reliably predict these strongly nonlinear flows remain limited. For turbulent flow interacting with very strong shocks, gas temperature increases dramatically after strong shocks. It is well known that thermal properties of air strongly depend on the temperature [28]. For example, at temperatures above 2000-2500 K, vibration energy mode is fully excited and $\mathrm{O}_{2}$ starts dissociating. Around $4000 \mathrm{~K}, \mathrm{O}_{2}$ is completely dissociated and $\mathrm{N}_{2}$ starts dissociating. Therefore, non-equilibrium flow effects including internal energy excitations, translation-vibration energy relaxation, and chemical reactions among different species need to be considered in DNS studies.

\section{AIMS AND OBJECTIVES}

Focus of our group at UCLA has been developing and using shock fitting methods as an alternative to complement the results and findings of shock capturing methods that are used by our other collaborators in the SciDAC project entitled "Simulations of turbulent flows with strong shocks and density variations". Developing high-order algorithms for shock and turbulence interactions and nonequilibrium flows is very challenging in itself. However, it has been observed that most widely used highorder shock capturing methods give low accuracy at the shock [29] and might lead to spurious oscillations [25]. Shock capturing methods rely on introducing appropriate numerical dissipation to avoid spurious oscillations which, however, is not accurate enough for simulation of turbulent flow. On the other hand, conventional high-order methods generally used for DNS studies have numerical problems due to strong gradients around shock. Thus, there is an inherent dilemma in using shock-capturing schemes for problems involving shocks as well as turbulence. While our other colleagues in this SciDAC project use advanced shock-capturing schemes to solve such problems, our group at UCLA explores utility of treating the shock sharply (as an internal boundary) to complement other approaches. Shock fitting algorithms treat the shock-interface sharply without any dissipation. Hence, they are compatible with low dissipation schemes used for DNS of turbulent flow. Shock/interface fitting methods are ideally suited for the cases where there is a clearly demarcated interface such as observed in the problems of isotropic turbulence interacting with shocks as shown in Fig. 1. Shock-fitting method can be easily combined with the shock-capturing methods to handle more complex problems.

Overall objective of current SciDAC project included first refining and development of numerical algorithms and then carry out benchmark DNS and LES of shock-turbulence interaction and the turbulent multi-material mixing of the planar and spherical Rayleigh-Taylor and Richtmyer-Meshkov instabilities. Our contributions to the science part of the SciDAC project are to develop and use shock-fitting methods as an alternative which treats the shock as a sharp entity, and to consider the real gas effects of nonequilibrium flow. During the assessment studies for the benchmark problems along with other SciDAC methods it was realized that shock fitting methods provide very accurate and reliable results for the 
problems where a well defined shock is present as in the case of shock turbulence interactions. Hence, our goals in the current SciDAC effort were identified as:

(i) Development and assessment of shock fitting methods for the problems of shock and turbulence interactions with capabilities to handle mutli-material mixing,

(ii) Conduct DNS studies of canonical shock-turbulence interaction problems, including analysis of the physics using the shock-fitting algorithms,

(iii) Development and assessment of shock fitting methods for non-equilibrium flow simulations using the the more realistic 11-species model of air and most recent thermo-chemical models.

Most promising shock-fitting methods from our tests in task 1 were implemented for interaction of shock turbulence interactions in the set up shown in Fig. 1. For these studies we generate inflow conditions as decaying isotropic turbulence fluctuations in separate computations and using the Taylor's hypothesis these fluctuations are convected with incoming flow to the shock. Postshock flow is then computed by shock fitting method. We have carried out a number of large DNS computations using this method for problem of shock and turbulence interactions. To complement the work being done by our collaborators (specifically Dr. J. Larsson) at Stanford, we have focused more on strong shocks for which shock-fitting method is more suitable. We have considered shocks as strong as Mach 30 in a number of configurations and found some new trends for very high Mach number flows while matching the trends observed by our collaborators at low Mach numbers. We have also made significant progress in the third goal of our objective. We have implemented to current shock-fitting code the more realistic 11-species model of air with most recent thermo-chemical models of non-equilibrium air flow. The non-equilibrium shock-fitting code can run cases with extremely high translational temperature.

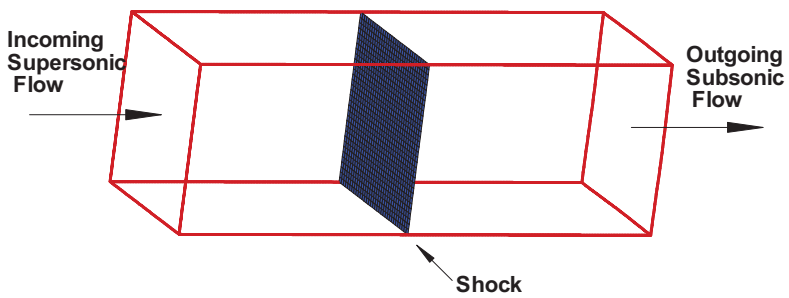

Fig. 1. A schematic of typical setting of isotropic shock and turbulence interaction.

\section{ACHIEVEMENTS}

During the period of the research project, we developed and implemented shock-fitting method for DNS study of interaction of isotropic turbulence with shock waves using perfect gas assumptions. A number of cases of different shock strengths, turbulent intensities and Reynolds number were considered. Moreover, we have also made significant progress in equipping these shock-fitting methods with nonequilibrium multi-species models. Main tasks achieved are as follows:

\section{A. Implementation of shock-fitting methods for interaction of isotropic turbulence and shocks.}

We have shown that the shock-fitting implementations that are uniformly high order accurate [3033] and suffer no spurious oscillations around the shock as generally observed with shock capturing formulations. We have implemented these shock-fitting methods for shock and isotropic turbulence interaction problems. The inflow conditions are generated in a separate simulation of decaying isotropic turbulence which ensures that turbulence fluctuations are realistic and follow fluid-flow equations. Using 
the Taylor's hypothesis these fluctuations are convected to the shock wave and shock fitting method is used to find the post shock flow. These implementations were validated by comparing the results against those available in the literature. We also applied for and were awarded approximately 6 million CPU hours at resources of NERSC and Teragrid/XSEDE. Using these large computer resources we have carried out more than 40 DNS computations with various inflow conditions. We have carried out DNS with mean Mach numbers of incoming flow ranging from 2 to 30. For the incoming flow, the turbulent Mach number, $M_{t}$, has been varied from 0.12 to 0.38 and Reynolds number based on Taylor microscale, $\operatorname{Re}_{\lambda}$ of upto 52.4 has been considered. We observe that some trends in turbulence statistics change at really high Mach numbers. We also observe that for sufficiently high Reynolds number of the flow, the vorticity fluctuations in post shock flow return to isotropy. However, return to isotropy is slower behind the stronger shocks. We also observe decrease in Taylor Microscales behind the shock for all cases. We will continue the computations of shock and turbulence interaction with perfect gas assumption. The results obtained from these studies will be used to find the relations between various input parameters and post shock turbulence statistics which can be used for developing further models.

\section{B. Development new shock-fitting code for computations of non-equilibrium flows}

Before shifting from SAP to the science part, we have been working on the SciDAC Science Application Partnerships in support of the "Turbulence" Science Application project of Stanford University. The goal is to provide novel numerical methods and related tools to simulate complex turbulent flows with strong shocks in an efficient, stable, accurate, and reliable manner. The main research achievements of UCLA research group are implementing the source terms of thermally nonequilibrium and chemically reactive flows, and backward Euler, SIRK3B and SIRK3C semi-implicit method to general shock-capturing schemes such as second-order TVD scheme and fifth-order WENO scheme. A basic two-temperature five-species model of air has been implemented and validated in SAP part. After that, we have also implemented to current shock-fitting code the more realistic 11-species model of air with most recent thermo-chemical models of non-equilibrium air flow, including gas-mixture viscosity, heat conductivity, and diffusion coefficients of Gupta et al. [34] and Yos [35], non-equilibrium reaction rates [36, 37], and equilibrium constants $[34,36,38]$. The non-equilibrium shock-fitting code can run cases with extremely high translational temperature. For example, the FIRE II reentry case has a peak translational temperature more than $30,000 \mathrm{~K}$.

\section{IMPLEMENTATION OF SHOCK-FITTING FOR SHOCK-TURBULENCE INTERACTION}

In our previous work we have developed and evaluated the shock-fitting methodology for canonical problems where flow disturbances interact with a strong shock [31, 32] in one and two dimensional setups. It was observed that results from shock-fitting methods for such problems showed uniformly high-order convergence and did not incur any spurious numerical oscillations. On the other hand, the popular shock-capturing methods were found to be only first order with non-physical oscillation around the shock. Since high-order accuracy is desired for the simulation of turbulent flow, shock-fitting methods provide a very good alternative to the shock-capturing method when a well defined strong shock is present in the flow. Hence, in this study we carry out simulation of flow with isotropic turbulence interacting with a strong well-defined shock using shock-fitting. Methodology and some of the shock turbulence results presented in this report are presented in more detail in Ref. [30]. 


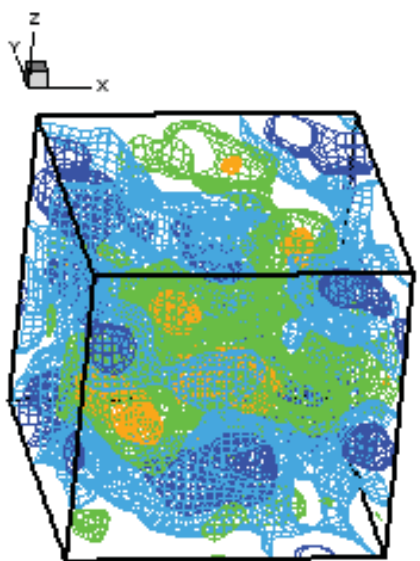

(a)

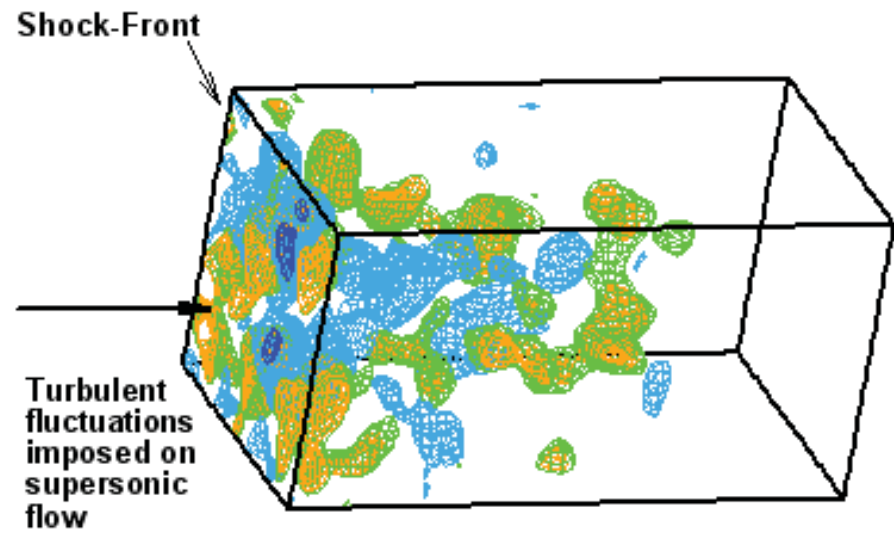

(b)

Fig. 2. Schematic for simulation of shock-turbulence interaction using shock-fitting algorithm. The inflow turbulence is generated by simulation of decaying isotropic turbulence in a periodic box as shown in (a). The turbulent fluctuations are imposed as left boundary condition of the actual computational domain for shockfitting algorithm as shown in (b).

With the shock-fitting algorithm for the problem shown in Fig. 1, there is no need to solve the supersonic flow upstream of the shock. Hence, computational domain for the shock-turbulence interaction consists of flow only downstream of the shock. The supersonic turbulent flow ahead of the shock can be computed in a separate simulation. Schematic of the shock-fitting implementation for the shockturbulence interaction problem is shown in Fig. 2. The inflow turbulence is generated using a separate direct numerical simulation as shown in Fig. 2(a). For the results presented in this abstract, we compute decaying isotropic turbulence in a periodic box to generate the realistic turbulent fluctuations that can be used as incoming turbulence for the shock-fitting algorithm. The computational domain for implementation of shock-fitting algorithm is shown in Fig. 2(b). The shock forms the left boundary of the computational domain. The turbulent fluctuations generated from Fig. 2(a) are imposed on supersonic flow and used as inflow condition at the shock. Periodic boundary conditions are used in the transverse directions and non-reflecting characteristic boundary conditions are used at the subsonic exit of the computational domain. In the shock-fitting algorithm, the grid distorts with movement of the shock the dimensions of the domain are time dependent.

\subsection{Inflow Conditions}

For the simulation of shock and turbulence interaction, turbulence in the flow just upstream of the shock should be realistic, fully developed and well characterized. Ideally, one should generate spatially evolving turbulence and prescribe it just behind the shock. However, it has been shown in the literature [39] that spatially evolving turbulent statistics can be approximated really well by those obtained from a temporal simulation using Taylor's hypothesis if the turbulent fluctuations are small enough. Based on this information, in this study, we generate the inflow conditions from temporal simulation in a periodic box and using Taylor's hypothesis the turbulent fluctuations are convected with mean shock-velocity as inflow condition for shock-fitting computation.

For simulation of decaying isotropic turbulence in a periodic box, initial conditions are generated using the algorithm given by Erlebacher et al [40]. The algorithm is based on generating random fields for fluctuations of flow variables and imposing a given spectrum. Following spectrum is imposed on the fluctuations of flow variables: 


$$
E(k) \sim k^{4} \exp \left[-2\left(k / k_{0}\right)^{2}\right]
$$

where $k=\sqrt{k_{1}^{2}+k_{2}^{2}+k_{3}^{2}}$ is the wave number of fluctuation and $k_{0}$ is the most energetic wave number.

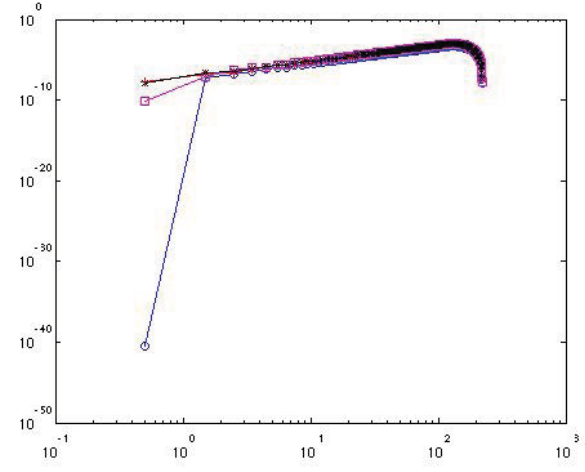

(a) random fluctuations

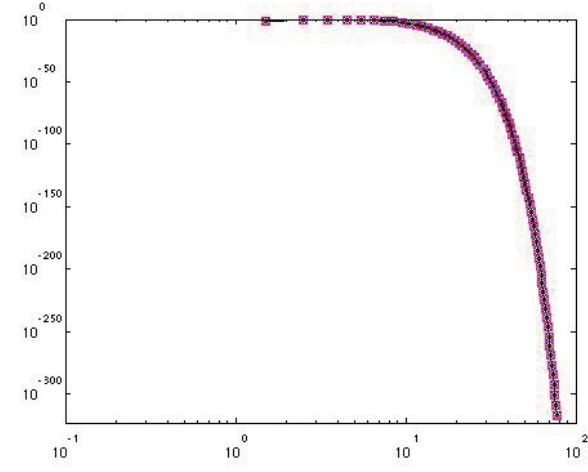

(b) scaled fluctuations

Fig. 3. Spectra of fluctuations of flow variables.

Figure 3 shows the energy spectra of fluctuations of flow variables before and after imposing the prescribed spectra. The fluctuation shown in Fig. 3(b) is used as initial conditions for the inflow simulation. This method offers flexibility to generate various turbulent regimes. The most important parameters that govern the physics of shock and turbulence interactions are turbulent Mach number $M_{t}$ and Reynolds number based on Taylor microscale $\operatorname{Re}_{\lambda}$.

The initial conditions are assigned in a box of dimension $(2 \pi)^{3}$ and compressible Navier-stokes equations are solved using periodic boundary conditions in all three directions until reasonably realistic turbulence is achieved. We use a fifth order upwind finite-differencing scheme [41] for this purpose. To validate our method of generating inflow turbulence, we have compared the results from our temporal simulation of decaying isotropic turbulence in a cubic box with those presented by Lee et al [39]. For this comparison the initial turbulence is generated with purely incompressible fluctuations with the spectrum given by (1) while density and pressure are kept at a constant value.

For the reference cases used for comparisons we have used streamwise Mach number $M_{1}=2.0$, fluctuation Mach Number $M_{t}=0.346$ and Reynolds number at inflow $\operatorname{Re}_{\lambda}=25.0$. Most energetic wave number $k_{0}=4$ is chosen. These quantities are defined as follows:

$$
\begin{aligned}
& M_{1}=\bar{u}_{1} / \bar{c} \\
& M_{t}=q / \bar{c}
\end{aligned}
$$

where,

$$
q=\left(\widetilde{u_{i}^{\prime \prime} u_{i}^{\prime}}\right)^{\frac{1}{2}}
$$

and

$$
\operatorname{Re}_{\lambda}=\bar{\rho} u_{r m s} \lambda / \bar{\mu}
$$

Here, for any given variable $f, \bar{f}$ denotes an ensemble average and $\tilde{f}$ is mass-weighted average i.e. $\tilde{f}=\overline{\rho f} / \bar{\rho}$. Deviation from ensemble average and mass-weighted average is is denoted as $f^{\prime}$ and 
$f^{\prime \prime}$ 'respectively. Subscript ' 1 ' has been used to denote the quantities upstream of the shock. Speed of sound is denoted as $c, u_{r m s}=\left(\widetilde{u_{1}^{\prime \prime 2}}\right)^{1 / 2}$ and Taylor microscale is $\lambda=\left(\lambda_{1}+\lambda_{2}+\lambda_{3}\right) / 3$ where

$$
\lambda_{\alpha}=\left[\overline{u_{\alpha}^{\prime 2}} / \overline{\left(\frac{\partial u_{\alpha}^{\prime}}{\partial x_{\alpha}}\right)^{2}}\right]^{1 / 2} \quad(\alpha=1,2 \text { or } 3)
$$

With the nondimensionalized governing equations following parameters are used as initial condition for generating initial random fluctuations: upstream mean density, $\rho_{1}=1$, temperature $T_{1}=1$, initial rms value of velocity fluctuations $u_{r m s}^{0}=1, \operatorname{Pr}=0.7, \gamma=1.4$. Any values of initial turbulent Mach number, $M_{t 0}$, and initial Reynolds number, $\operatorname{Re}_{\lambda 0}$ are can be chosen. Nodimensionalized gas constant is given by $R=3 / \gamma M_{t, 0}^{2}$ and reference viscosity is given as $\mu_{0}=\rho_{1} u_{r m s}^{0} \lambda_{0} / \operatorname{Re}_{\lambda, 0} \quad \lambda_{0}=2 / k_{0}$.

The results obtained from the method described above are compared with those obtained from Lee et al [39] in Fig. 4. The variable used for comparison is skewness of streamwise velocity which is defined as:

$$
S_{1}=\overline{\left(\partial u_{1}^{\prime} / \partial x_{1}\right)^{3}} / \overline{\left[\left(\partial u_{1}^{\prime} / \partial x_{1}\right)^{2}\right]^{3 / 2}}
$$

Skewness of velocity derivatives is a measure of intertial non-linearity of turbulence. For the parameters considered here, a realistic turbulence should have $S_{1}$ in the range -0.4 to $-0.6[8,23,24]$. From Fig. 4, it can be observed that even though started from random initial flow-fields, evolution of skewness of streamwise velocity obtained from the method described in this section matches well to those mentioned in the literature. Also, realistic turbulence is obtained around and after $t / \tau_{t}=0.7$. It should be noted that results by Lee et al [39] were for a set of $64^{3}$ grid-points and show a very good match to our results from same number of grid-points. It indicates that the inflow conditions generated from our computations are good approximations of the realistic turbulence required for specifying inflow boundary conditions.

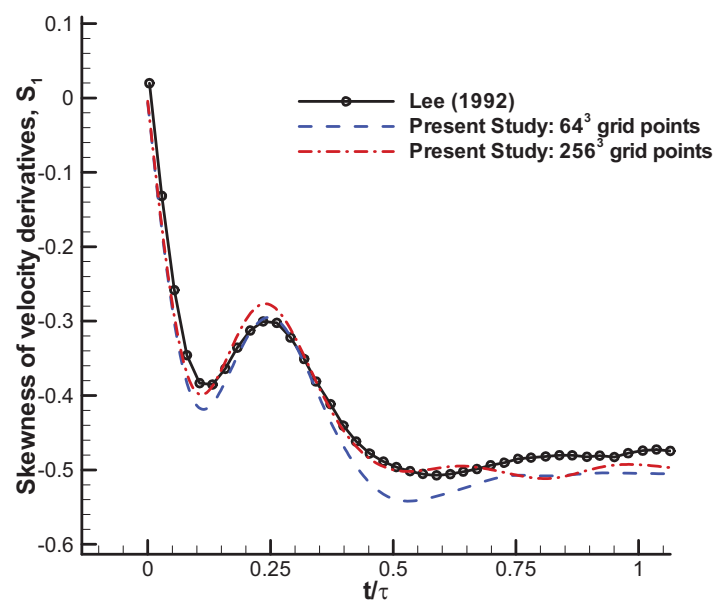

Fig. 4. Comparison of evolution of velocity derivative skewness obtained from the $5^{\text {th }}$ order upwind method used in this study with those available from literature $\left(M_{t}=0.346, \operatorname{Re}_{\lambda}=25.0\right.$ and $\left.\tau_{t}=\lambda / u_{\text {rms }}\right)$ 
Inflow conditions can be generated for various values of $M_{t 0}$ and $\mathrm{Re}_{\lambda 0}$ as desired in the shock fitting simulations. For example, the temporal simulation was started with $M_{t 0}=0.2$ and $\operatorname{Re}_{\lambda 0}=10.0$ and values generated at $u_{r m s} t / \lambda=1.0$ were used as upstream condition for the shock fitting simulations. This corresponds to $M_{t, u}=0.121, S_{1, u}=-0.45$ and $\mathrm{Re}_{\lambda, u}=6.18$ (Here, subscript 'u' denotes upstream values for the shock fitting computation.) Following four cases of upstream values are presented in this draft:

Case I: $M_{t, u}=0.121, S_{1, u}=-0.45$ and $\operatorname{Re}_{\lambda, u}=6.8$

Case II: $M_{t, u}=0.126, S_{1, u}=-0.46$ and $\operatorname{Re}_{\lambda, u}=15.8$

Case III: $M_{t, u}=0.185, S_{1, u}=-0.52$ and $\operatorname{Re}_{\lambda, u}=39.7$

Case IV: $M_{t, u}=0.275, S_{1, u}=-0.47$ and $\operatorname{Re}_{\lambda, u}=22.4$

Case V: $M_{t, u}=0.376, S_{1, u}=-0.46$ and $\operatorname{Re}_{\lambda, u}=39.7$

For the parameters considered here, a realistic turbulence should have $S_{1}$ in the range -0.4 to - 0.6 $[8,23,24]$. In all of our calculations of inflow turbulence we found that $S_{1}$ reaches steady state in $t \sim \lambda_{0} / u_{r m s}^{0}$. Figure 5 shows variations of various statistics obtained from simulations for flow with initial parameters $M_{t 0}=0.175$ and $\operatorname{Re}_{\lambda 0}=135$, and $M_{t 0}=0.15$ and $\operatorname{Re}_{\lambda 0}=50$, respectively. These computations were performed with $256^{3}$ grid points. Apart from $S_{1}$, we also plot turbulent Mach number, $M_{t}$, variance of velocity fluctuations, Reynolds number based on Taylor microscale, $\mathrm{Re}_{\lambda}$, and variance of dilatation fluctuations, $d=\partial u_{i} / \partial x_{i}$. It can be seen that velocity fluctuations are dissipated with the time, leading to decay in turbulent Mach number as well as Taylor microscale. Sudden increase in dilatation is due to completely solenoidal initial conditions and has been reported in previous studies as well $[42,43]$.

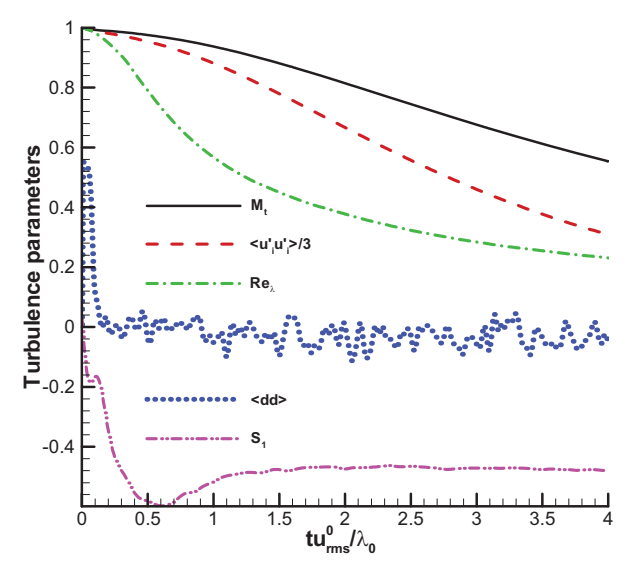

(a) $M_{t 0}=0.175, \operatorname{Re}_{\lambda 0}=135$

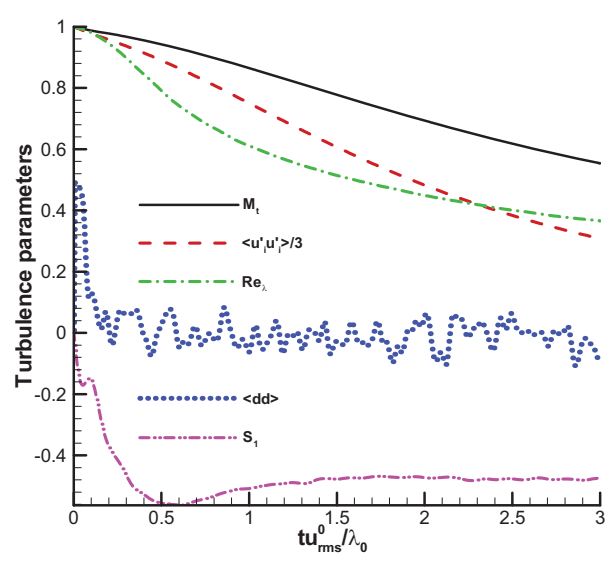

(b) $M_{t 0}=0.15, \operatorname{Re}_{\lambda 0}=50$

Fig. 5. Variation of various turbulence statistics in simulation of decaying isotropic turbulence.

After the skewness of velocity derivative, $S_{1}$, becomes steady to have a value between - 0.4 and 0.6 , we choose a flow-field with desired values of $M_{t}$ and $\operatorname{Re}_{\lambda}$ as inflow condition for the shock-fitting 
computations. One can vary the flow conditions of decaying isotropic turbulence to obtain well developed realistic turbulence with desired statistical properties.

We further compute 4 series of DNS computations with varying incoming flow of turbulence intensities $M_{t}$ from 0.083 to 0.143 , mean Mach number from 2 to 30 , and Reynolds number, $\operatorname{Re}_{\lambda}$, from 18.9 to 52.4. Inflow conditions of Cases VI \& VII are obtained from the decaying isotropic turbulence computation with initial parameters $M_{t 0}=0.175$ and $\operatorname{Re}_{\lambda 0}=135$ at $t u_{r m s}^{0} / \lambda_{0}=2.0$ and 3.0 as shown in Fig. 5(a). Whereas inflow conditions of Cases VIII \& IX are obtained from the decaying isotropic turbulence computation with initial parameters $M_{t 0}=0.15$ and $\operatorname{Re}_{\lambda 0}=50$ at $t u_{r m s}^{0} / \lambda_{0}=2.0$ and 3.0 as shown in Fig. 5(b).

Table 1. Four cases of inflow conditions used in DNS of shock and turbulence interaction

\begin{tabular}{|c||c|c||c|c|}
\hline & $\mathrm{M}_{1}$ & $\mathrm{M}_{\mathrm{t}}$ & $\mathrm{Re} \lambda$ & Grids \\
\hline \hline Case VI & $2-30$ & 0.143 & 52.4 & $256 \times 256 \times 512$ \\
\hline Case VII & $2-30$ & 0.118 & 39.4 & $256 \times 256 \times 512$ \\
\hline \hline Case VIII & $2-30$ & 0.104 & 23.1 & $256 \times 256 \times 512$ \\
\hline Case IX & $2-30$ & 0.083 & 18.9 & $256 \times 256 \times 512$ \\
\hline
\end{tabular}

The computational domain for DNS of shock and turbulence interaction is schematically shown in Fig. 2(b). The shock forms the left boundary of the computational domain. The turbulent fluctuations generated from Fig. 2(a) on a periodic box of dimensions $2 \pi^{3}$ are imposed on supersonic flow and used as inflow condition at the shock. For shock-fitting computations, we use a domain of size $4 \pi \times 2 \pi^{2}$ and same non-dimensionalization of flow variables is used. Uniform conditions corresponding to laminar Rankine-Hugoniot jump conditions are used as initial condition for simulation of post-shock flow. As the shock interacts with the incoming turbulence, transient features are generated. Several flow-through of inflow box are needed before turbulence statistics in post-shock flow reach a steady state.

It is observed from previous shock-turbulence interaction simulations that turbulent fluctuations are generally much stronger just behind the shock. Hence, it is advisable to cluster more grid points near the shock. The grid-spacing in transverse direction is determined by the lengthscales in DNS of turbulent flow. For simulation of isotropic flows, it has been suggested that one should resolve a wavelength of $4.5 \eta_{s}$ where $\eta_{s}$ is the Kolmogorov length scale for the flow in the computational domain [44]. With our fifth-order finite-difference scheme such resolution will require a grid spacing of $2.0 \eta_{s}$ in transverse direction. On the upstream side of the shock, the Kolmogorov length scale is defined as $\eta_{0} \approx 0.51 \lambda / \sqrt{\operatorname{Re}_{\lambda}}$. Larsson and Lele [45] have recently presented a relation for the change in the Kolmogorov length scale across the shock which leads to $\eta_{s} \approx \eta_{0}\left(\rho_{s} / \rho_{u}\right)^{-11 / 8}\left(p_{s} / p_{u}\right)^{3 / 8}$ [27]. With $\lambda \approx 2 / k_{0}$, about $6.1 k_{0} \sqrt{\operatorname{Re}_{\lambda}}\left(\rho_{s} / \rho_{u}\right)^{11 / 8}\left(p_{s} / p_{u}\right)^{-3 / 8}$ grids are needed in transverse directions. Based on these requirements, we chose to use 256 grid points in transverse direction.

For the computations of statistics, we found that storing and computing averages from 60 instantaneous flow-fields during time interval $T$ is necessary for statistical convergence, where $T$ represents the time needed for the inflow passing through one length of periodic box. 


\subsection{Shock and Turbulence Interaction}

Turbulent fluctuations described in the previous section are imposed on corresponding supersonic flow following Taylor's hypothesis that is valid for small turbulent intensities $\left(M_{t}<0.5\right.$ and $\left.u_{1 r m s}^{\prime} s \bar{u}_{1}<0.15\right)$. For higher turbulent intensities, it is advisable to carry out simulation of spatially decaying turbulence which is more expensive. From the temporal simulations inside a periodic box, we obtain values of flow variables at fixed grid points of the box while due to shock-movement grid points in shock-fitting computations are not stationary. Moreover, when the turbulent box is convected through the shock in the shock-fitting computations, the shock-points generally do not align with grid points of the turbulent box. Hence, values on the supersonic side of the shock are computed using interpolations. Since in our shock-fitting formulation the grids move in only one direction (X-direction in Fig. 2(b)), one dimensional interpolation using fast fourier transform (FFT) is sufficient for this purpose. As a boundary condition, shock-fitting formulation needs the values of the time derivatives of conservative variables ahead of the shock according to the isotropic field which using Taylor's hypothesis are taken as appropriate spatial derivatives. Together with one characteristic coming to the shock from the high pressure side, these values determine the shock velocity. Thus, downstream flow variables are calculated from the corresponding upstream values, using the Rankine-Hugoniot conditions.

DNS of the shock and turbulence interaction needs a large number of grids to fully resolve all the scales involved. For simulation of isotropic flows, it has been suggested [44] that one should resolve a wavelength of $4.5 \eta_{s}$ where $\eta_{s}$ is the Kolmogorov length scale for the flow in the computational domain. With our high order finite difference scheme such resolution will require a grid spacing of $2.0 \eta_{s}$ in transverse direction. On the upstream side of the shock, the Kolmogorov length scale is defined as $\eta_{0} \approx 0.51 \lambda / \sqrt{\operatorname{Re}_{\lambda}}$. Larsson and Lele have recently presented the relation for change in Kolmogorov length scale across the shock which leads to $\eta_{s} \approx \eta_{0}\left(\rho_{s} / \rho_{u}\right)^{-11 / 8}\left(p_{s} / p_{u}\right)^{-11 / 8}$ [27]. Assuming $\lambda \approx 2 / k_{0}$, approximately $N>\frac{6.1 k_{0} \sqrt{\operatorname{Re}_{\lambda}}}{\left(\rho_{s} / \rho_{u}\right)^{-11 / 8}\left(p_{s} / p_{u}\right)^{-11 / 8}}$ grids are needed in all directions. Based on these requirements, we chose to use 128 grid points in transverse direction for the inflow Reynolds number of $\operatorname{Re}_{\lambda, u}=6.8$ and Mach number $M_{1}=2-20$. While 256 grid points in transverse direction were found to be sufficient for $\operatorname{Re}_{\lambda, u}=39.7$ and $M_{1}=2-20$.

While computing the flow for shock-turbulence interaction problems considered here, it was observed that turbulent intensity is generally higher just behind the shock. A typical distribution of density values in X-Y plane is shown in Fig. 6(a). Regions of highest gradients are observed just behind the shock while fluctuations attenuate moving towards the exit. Hence, to appropriately resolve the flow it is advisable to stretch the grid to cluster it near the shock wave). After numerical experiments, 512 grids in streamwise direction were found to be sufficient while the grids were stretched in streamwise direction such that near the shock $\Delta x_{2} / \Delta x_{1} \approx 18$. At the exit, non-reflecting boundary conditions of Poinsot and Lele [46] are used. Also, one-fourth of computational grid points near outflow is used as sponge zone to damp out the acoustic reflections from the exit boundary. To ensure the correct implementation of boundary conditions, we show in Fig. 6(b) instantaneous pressure variation along centerline of the domain using two different domain lengths and using same grid spacing. It can be observed that boundary conditions used for domain $4 \pi \times 2 \pi^{2}$ ensure that the results are same as those produced by taking a longer domain. It was observed that after two flow-through of the periodic isotropic turbulence through the shock, statistically steady state is established in the computational domain. Based on our numerical experiments for statistical convergence, for computation of turbulence statistics, we save and use 60 instantaneous fields per flow-through time. 


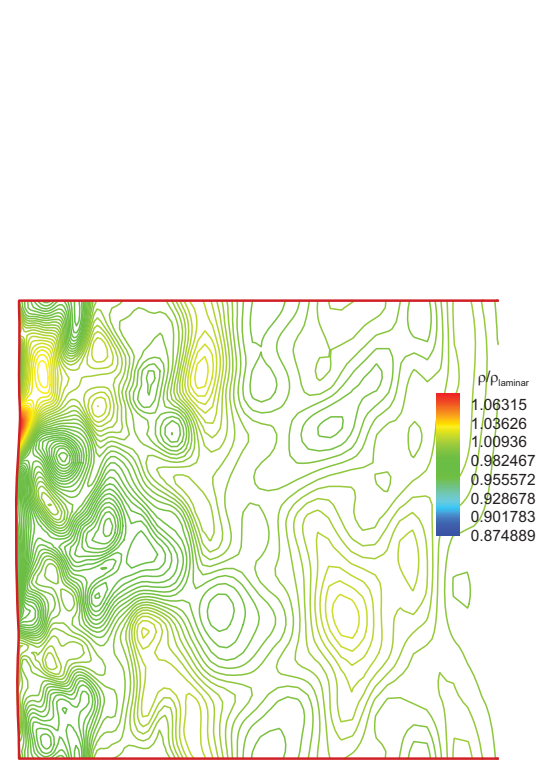

(a)

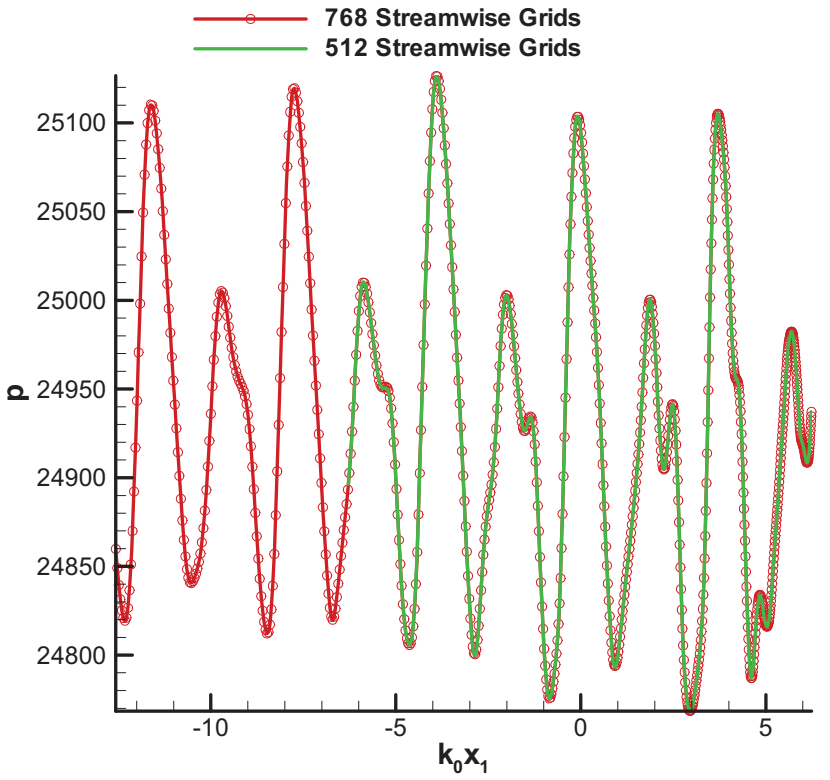

(b)

Fig. 6. (a) Instantaneous iso-contours of density in $X-Y$ plane at the top plane $(Z=2 \pi)$ (b) Instantaneous pressure profile in streamwise direction along the center line of the domain after 8 flow-through times for $M_{1}=20$ flow for case III using two different domain lengths.

\section{RESULTS FOR SHOCK TURBULENCE INTERACTIONS}

As discussed earlier, there are not many studies available in the literature which consider strong shocks for the shock turbulence interactions. Compared to the commonly used shock capturing methods, shock fitting methods are more suitable for simulations with very strong shocks. In the present study, we have varied the upstream Mean mach number, $M_{1}$ from 2 to 20 while imposing inflow fluctuations of Cases I to $\mathrm{V}$ as discussed in Section 4.1. Some of the basic results are presented in this section and has been compared with previous numerical and theoretical studies.

\subsection{Mean Profiles}

Profiles of mean density and streamwise velocities are shown in the Fig. 7 for inflow conditions of case II. Some results for inflow of case I were presented in our previous study [30]. It is observed that the jump in the mean turbulent density and pressure is smaller than that observed in the laminar flow. On the other hand jumps in mean velocity value are higher than that predicted by laminar Rankine Hugoniot jump conditions for all the Mach numbers considered in this study. This is consistent with the prediction Rapid Distortion Theory (RDT) of Lele [47]. It is also observed that as Mach number is increased the deviation of turbulent and laminar means also reduces. This is also expected since turbulent Mach number of the inflow is kept constant leading to lower turbulent intensity $\left(u_{r m s} / \bar{U}\right)$ values. With the passage of turbulence, shock wave gets distorted. Variation of rms values of shock fluctuations with Mach number is presented in Fig. 7(d). It is again observed that smaller turbulent intensities (for higher Mach number flows) lead to smaller fluctuations in the mean shock profiles. Actually it was observed that fluctuations in the shock location values $\propto 1 /\left(M_{1}^{2}-1\right)$. Although not shown here, we also observe that higher 
turbulence Mach number, $M_{t}$ leads to higher fluctuations in the shock locations (Comparing cases III and $\mathrm{V})$.

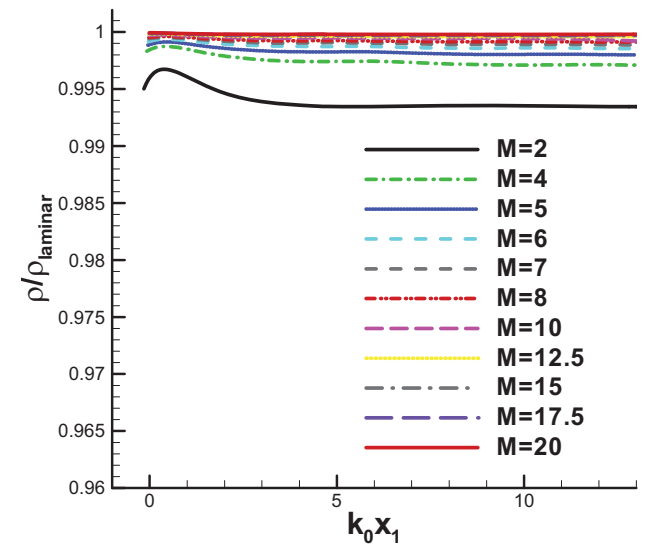

(a)

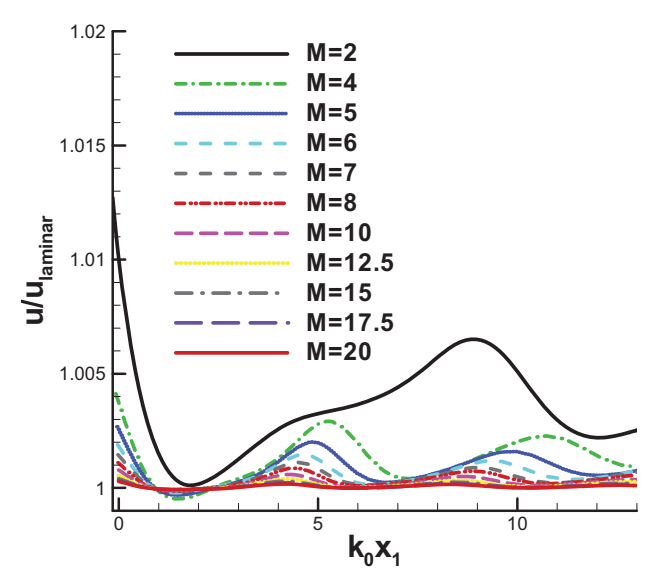

(c)

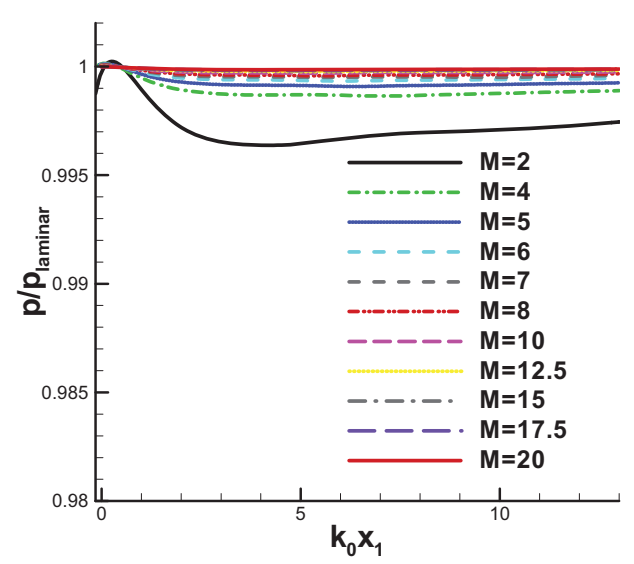

(b)

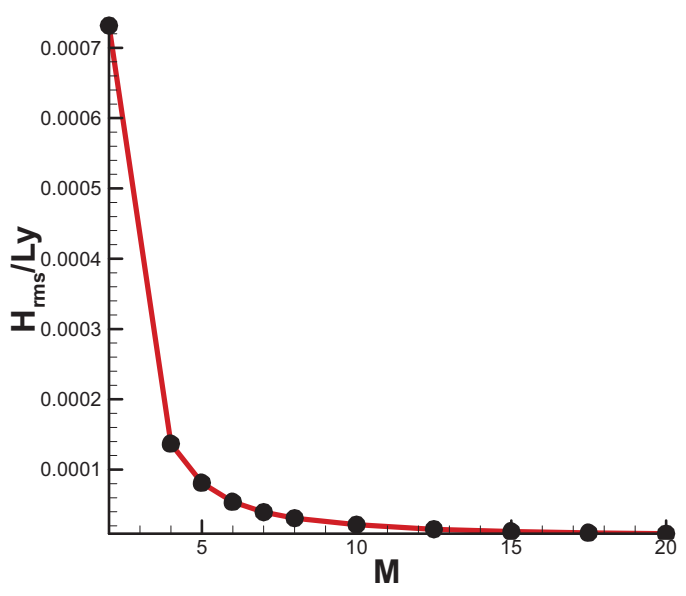

(d)

Fig. 7. Profile of (a) mean density (b) pressure and (c) mean velocity in streamwise direction for $\operatorname{Re}_{\lambda}=15.8, M_{t}=0.126$. All the values are normalized by corresponding values in the laminar flow. (d) Variation of rms value of shock distortion with Mach number (normalized with transverse length of the computational domain).

\subsection{Reynolds Stressess}

Linear interaction analysis (LIA) of shock turbulence interactions considered by various researchers $[48,49]$ show that turbulent velocity fluctuations are amplified as they move across the shocks. Moreover, the fluctuations in velocity components are significantly affected by the evanescent acoustic waves across the shocks. LIA results for evolution of the normal components of Favre's Reynolds stresses, $R_{i j}=\widetilde{u_{i}^{\prime \prime} u_{j}^{\prime \prime}}$, behind the shock wave is plotted in Fig. 8 for $M_{1}=2.0$ (as presented by Lee et al. [23]). These values are also compared against those obtained from out shock fitting method for Case I $\left(M_{1}=2.0, \operatorname{Re}_{\lambda}=6.18\right.$ and $\left.M_{t}=0.12\right)$. All the stresses are normalized by the upstream Reynolds stresses. $x_{1}=0$ denotes the mean position of the shock. 


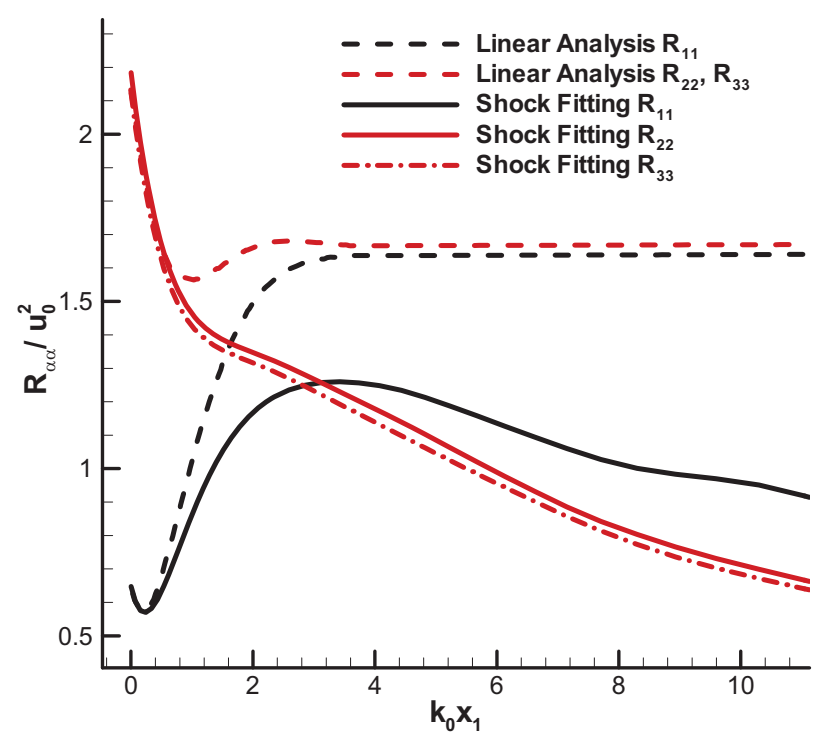

Fig. 8. Evolution of diagonal components of the Reynolds Stress tensor $R_{i j}=\widetilde{u_{i}^{\prime \prime} u_{j}^{\prime \prime}} / u_{0, u}^{2}$ behind the shock compared against those obtained from Linear Interaction analysis [23, 49].

It is observed that the turbulent fluctuations in the streamwise directions are reduced immediately behind the shock while the fluctuations in the transverse velocity increase. Just behind the shock, the computed amplification of Reynolds stresses match well to those obtained from LIA. However, in the far field LIA results and computed results differ significantly as viscous decay of stresses was not taken into account in LIA. For both the LIA and computed results, the streamwise fluctuations rapidly evolve behind the shock until a length of $\sim 3 / k_{0}$. This can be attributed mainly to the contribution of evanescent acoustic waves to the streamwise velocity just behind the shock. Maximum streamwise Reynolds stresses reached behind the shock in the computed flow are significantly smaller than those predicted by LIA.

For low turbulence intensities linear theory was found to predict the values of streamwise Reynolds stress just behind the shock very well. However, the far field is significantly affected by the viscous dissipation. Effect of increasing Mach number on streamwise Reynolds stresses is presented in Fig. 9 for three inflow conditions with low turbulence fluctuations $\left(M_{t}<0.2\right)$. It can be observed that with change in Mach number the streamwise Reynolds number changes profile behind the shock. It is observed that the profile of streamwise Reynolds number shows two peaks behind the shock before dissipating continuously in farfield. As can be seen from Fig. 9(a),(b) and (c), for Mach 2 inflow, we see that the maximum streamwise Reynolds stress occurs at the maxima close to the shock wave. However, as we increase the Mach number of the incoming flow, streamwise Reynolds increases downstream of the first maxima. For very strong shocks we clearly see that maximum variance of streamwise velocity fluctuations is obtained at the second peak. For an estimate of how the amplification of streamwise velocity is affected by change of Mach number and Reynolds numbers, we have plotted these amplifications for cases I, II and III and compared them with Linear theory results of Lee [23] and results from our collaborators (Dr. Larsson and Prof. Lele) in the current SciDAC effort [45]. As done by Larsson et al., we also extrapolate the farfield value to the mean shock location for this comparison. We see that with increase in Reynolds number of the incoming flow, the streamwise Reynolds stress values are amplified more due to reduction in viscosity values. Actually for similar Reynolds number of the 
incoming flow ( $\operatorname{Re}_{\lambda} \approx 40$ ), values obtained from the shock fitting computations match very well to those obtained by our collaborators. It is interesting to observe that as we increase the Mach number of the incoming flow, we see that amplification in streamwise Reynolds stress first reduces and then increases. It should be noted such high Mach number calculations have not been considered in the past. While previous studies of Ref. [23] and [45] suggested reduction in the streamwise Reynolds stress with increase in Mach number of the incoming flow, we see that it is not true for very strong shocks. We also computed relatively high turbulent Mach number flows using our shock-fitting method and observe that deviation from linear theory results is significant. Such cases are Case IV and Case V. Results for these cases are presented in Fig. 10. It is observed that for such cases streamwise fluctuations continuosly increase with increase in Mach numbers.

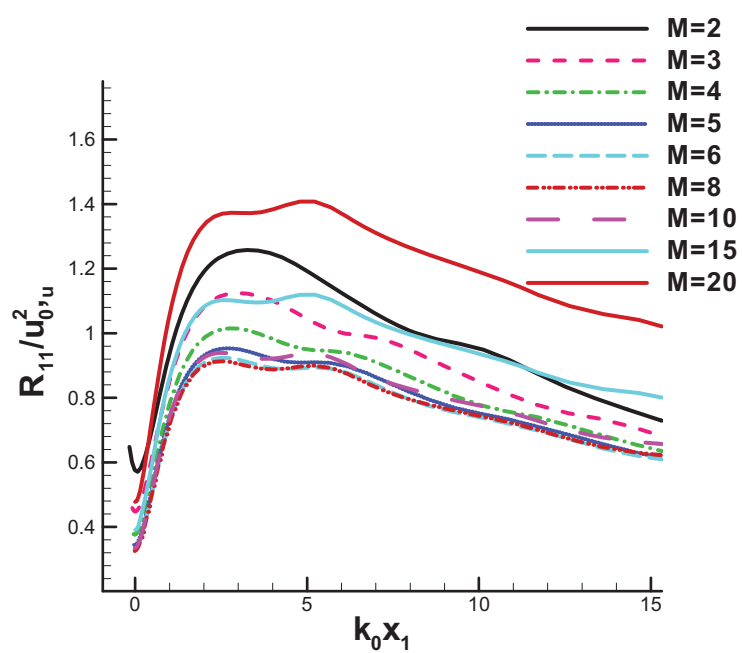

(a) $\operatorname{Re}_{\lambda}=6.8, M_{t}=0.121$

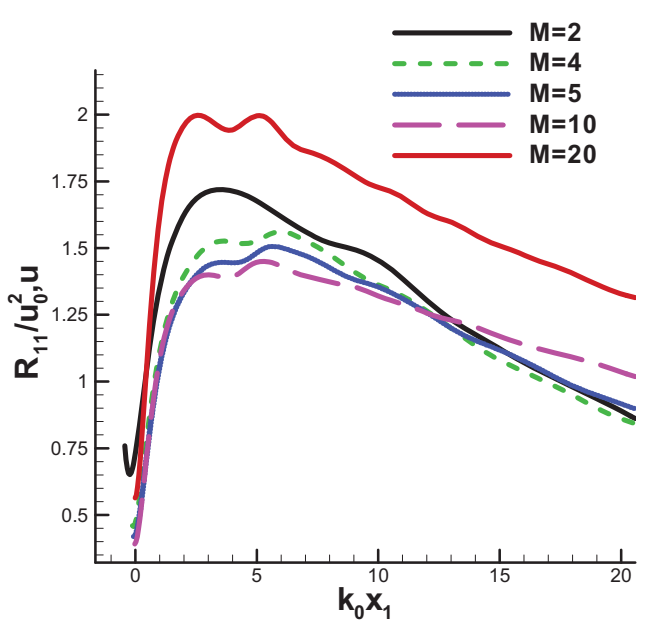

(c) $\operatorname{Re}_{\lambda}=39.7, M_{t}=0.185$

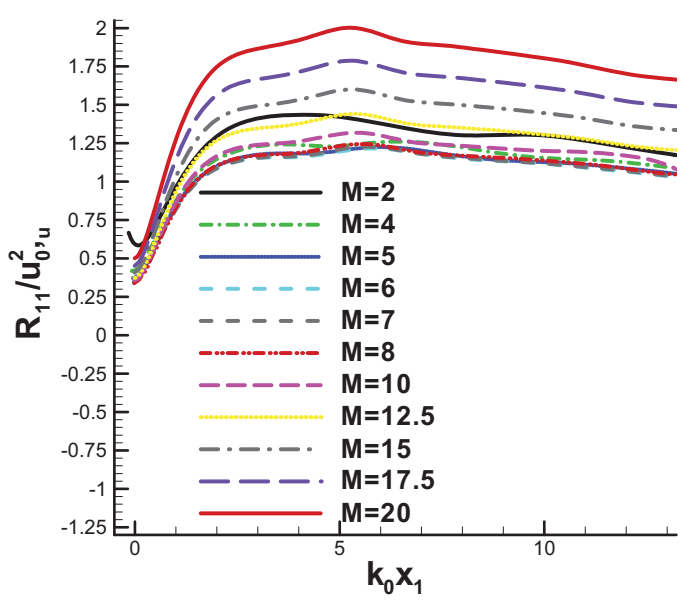

(b) $\operatorname{Re}_{\lambda}=15.8, M_{t}=0.126$

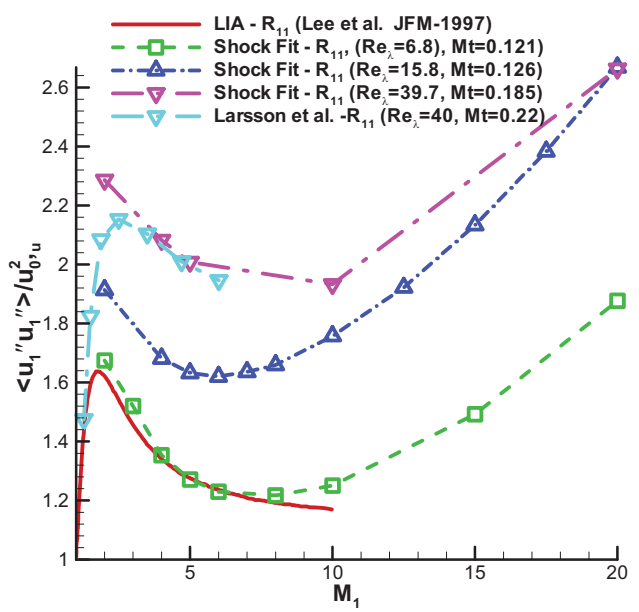

(d) Variation of amplification of $R \& M_{t}$

Fig. 9. Amplification in streamwise velocity fluctuations for three different cases of Reynolds numbers and turubulence Mach numbers: (a) $\operatorname{Re}_{\lambda}=6.8, M_{t}=0.121$ (b) $\operatorname{Re}_{\lambda}=15.8, M_{t}=0.126$ and (c)

$\operatorname{Re}_{\lambda}=39.7, M_{t}=0.185$.(d) Variation of amplification of streamwise Reynolds number with shock strength (results have been extrapolated from far field to the mean shock position). 


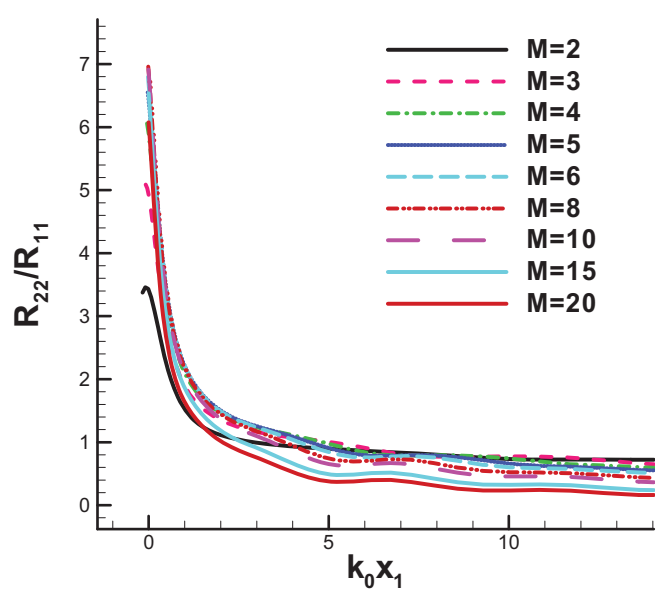

(a) $\operatorname{Re}_{\lambda}=6.8, M_{t}=0.121$

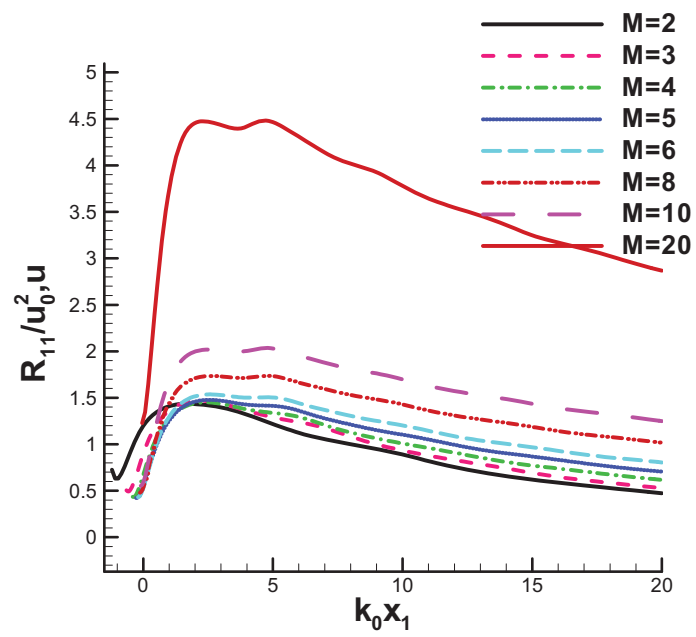

(c) $\operatorname{Re}_{\lambda}=22.4, M_{t}=0.275$

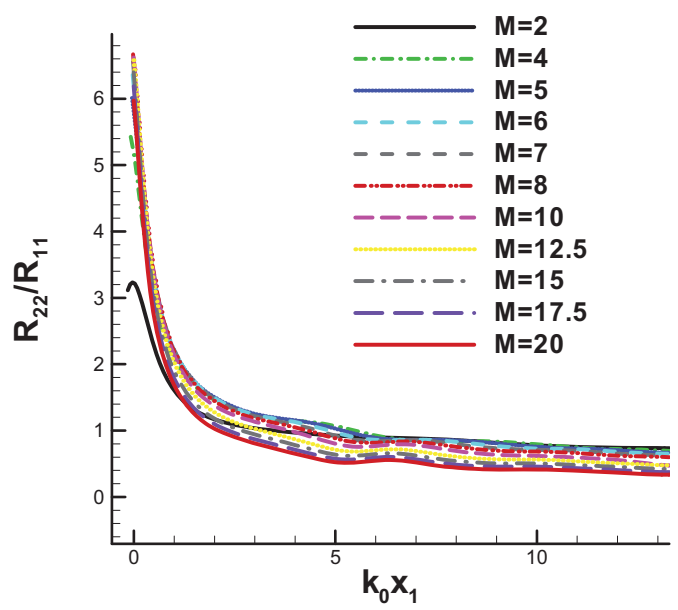

(b) $\operatorname{Re}_{\lambda}=15.8, M_{t}=0.126$

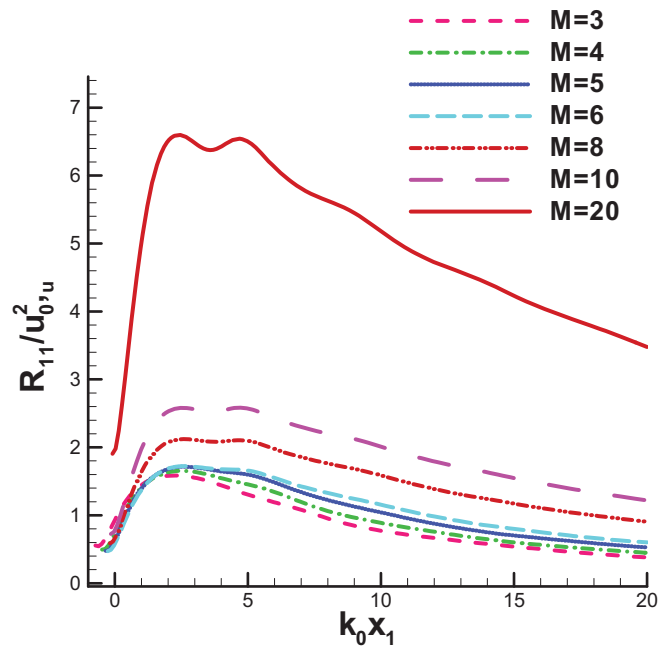

(d) $\operatorname{Re}_{\lambda}=39.7, M_{t}=0.376$

Fig. 10. Amplification in streamwise velocity fluctuations for high turubulence Mach numbers.

It was found that for all the cases of Mach number considered, $R_{11}$ is always smaller than $R_{22}$ downstream of the shock. Anisotropy in the velocity variances is quantified by the ratio $R_{22} / R_{11}$ which is plotted in the Fig. 11. It is observed that this ratio continuously decreases downstream of the shock and there is no evidence of the flow returning to isotropy. For higher Reynolds number flows, decay in velocity fluctuations is slower due to smaller viscous dissipation. 


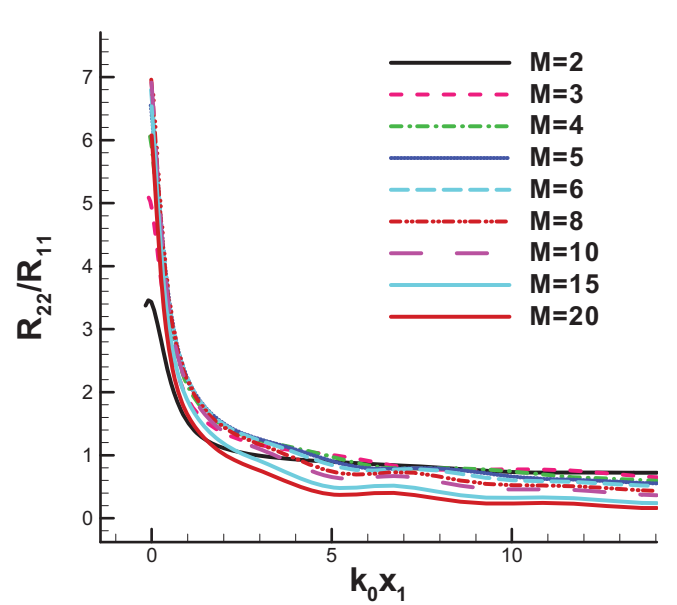

(a) $\operatorname{Re}_{\lambda}=6.8, M_{t}=0.121$

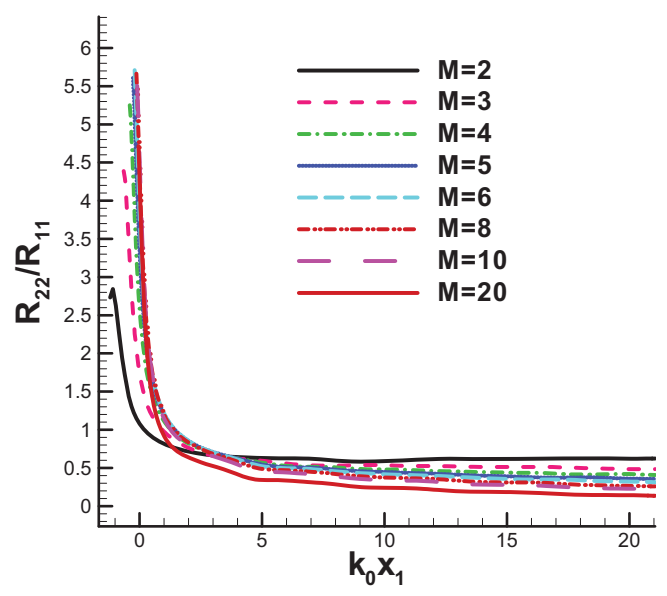

(c ) $\operatorname{Re}_{\lambda}=22.4, M_{t}=0.275$

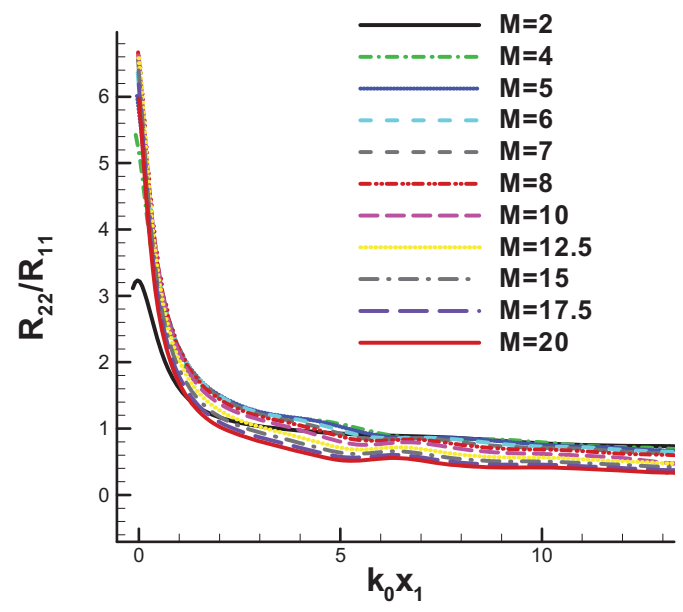

(b) $\operatorname{Re}_{\lambda}=15.8, M_{t}=0.126$

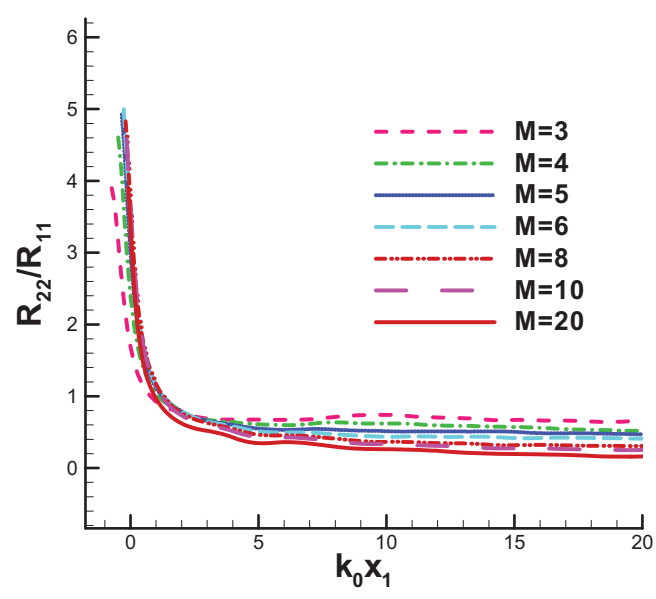

(d) $\operatorname{Re}_{\lambda}=39.7, M_{t}=0.376$

Fig. 11. Anisotropy in velocity fluctuations in post-shock flow for interaction of shock with isotropic turbulence for four different cases of Reynolds numbers and turubulence Mach numbers.

Streamwise variation of $R_{11}$ for various shock strengths is shown in Fig. 12 for inflow conditions of case VI. In general, the $R_{11}$ values evolve rapidly behind the shock for all the shock strengths considered and reach maximum value before $x_{1}=10 / k_{0}$. It is observed that maximum amplification of Reynolds stress $\mathrm{R}_{11}$ decreases as the Mach number of the mean flow is increased till 8.8. The decrease in $R_{11}$ is consistent with findings of linear interaction analysis. This trend, however, reverses as shock strength is increased beyond Mach 8.8. For stronger than Mach 8.8 shocks, the Reynolds stress $R_{11}$ is amplified as mean Mach number is increased. Similar conclusion can be drawn in Fig. 13, where streamwise variation of $R_{11}$ for various shock strengths for inflow conditions of case VIII is shown. The 
only difference between Figs. 12 and 13 is the magnitude of Reynolds stress. Due to the weaker freestream isotropic turbulence, the magnitude of Reynolds stress in Fig. 13 is smaller than its counterpart in Fig. 12.
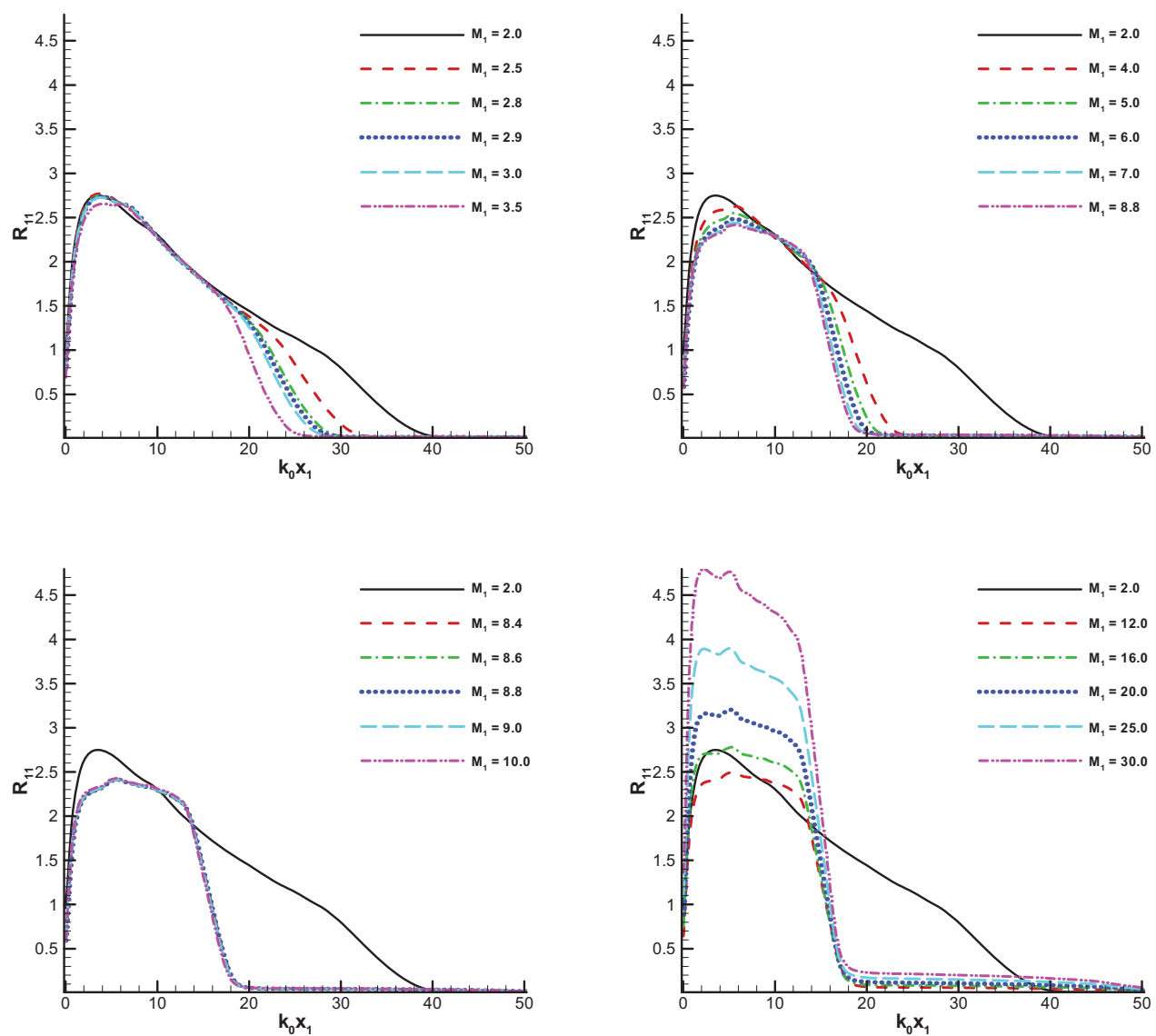

Fig. 12. Evolutions in streamwise-streamwise Reynolds stresses for inflow conditions of case VI.

The results of case VII are quite similar to those of case VI. And the results of case IX are quite similar to those of case IX. Because of the decrease of freestream isotropic turbulence from case VI to case IX, streamwise-streamwise Reynolds stresses of case VII and case IX are lower than those of case VI and case VIII, respectively.

\subsection{Vorticity variance}

For the quasi-incompressible inflow turbulence considered in this study, one of the most important contributions to the dissipation of turbulent kinetic energy is expected from the vorticity fluctuations. For the cases I, II, IV, V, the evolution of streamwise vorticity fluctuations, $\widetilde{\omega_{1}^{\prime \prime} \omega_{1}^{\prime \prime}}$ behind the shock is presented in the Fig. 14. The values are normalized by the value of vorticity variance, $\omega_{0}^{2}=\widetilde{\omega_{i}^{\prime \prime} \omega_{i}^{\prime \prime}} / 3.0$, in the isotropic flow just ahead of the shock. It can be seen that the streamwise vorticity 
fluctuations do not change significantly as the flow passes through the shock. This is in accordance with the linear theory and results obtained in previous studies $[6,17,23,24]$. On the other hand, transverse vorticity fluctuations are significantly amplified just behind the shock.
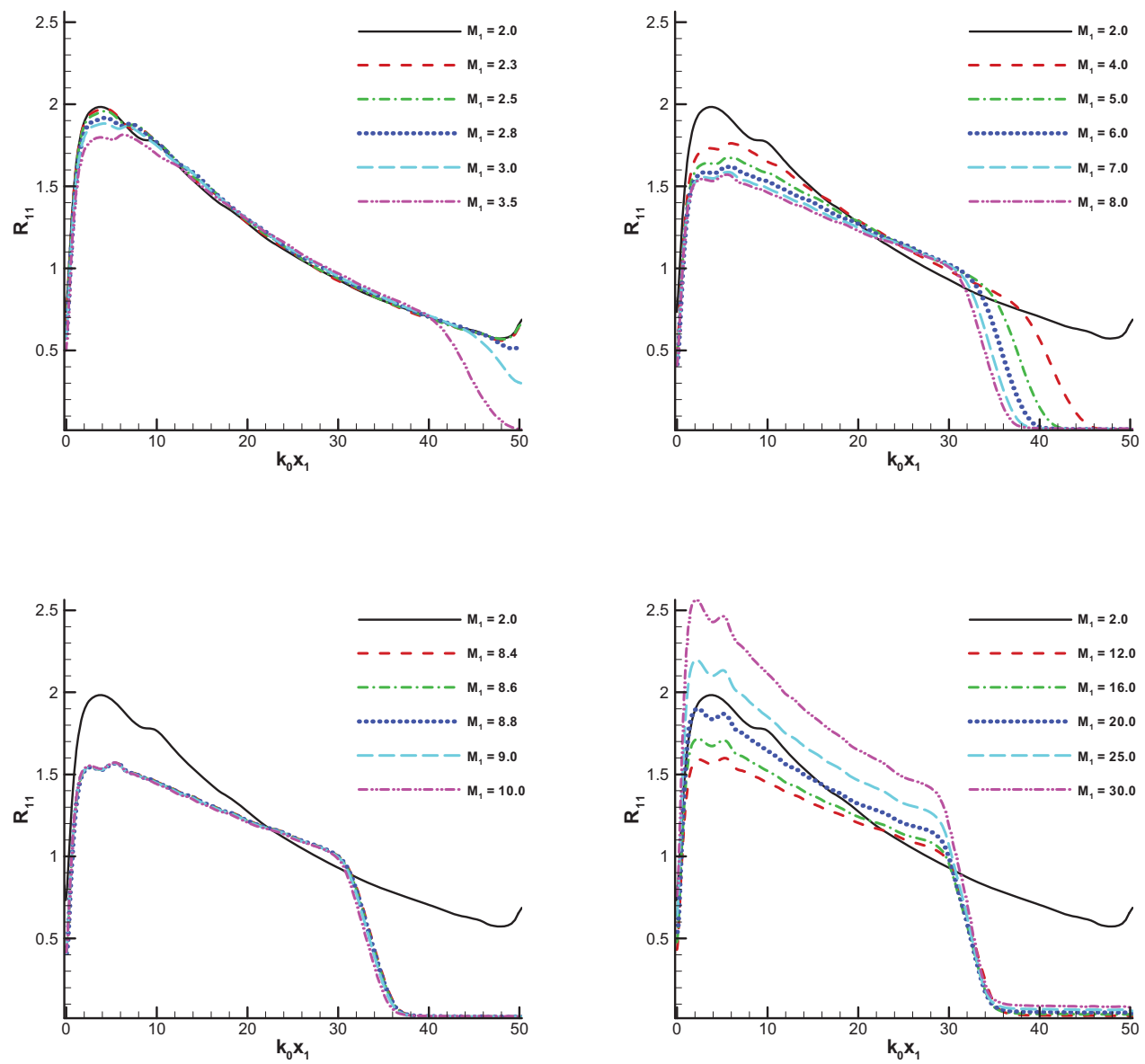

Fig. 13 Evolutions in streamwise-streamwise Reynolds stresses for inflow conditions of case VIII.

For inflow conditions of case I, all the vorticity variances were seen to decay behind the shock. However, as Reynolds number is increased a small increase in the streamwise vorticity is seen downstream of the shock which is attributed to the non-linear tilting and stretching of vorticity. Such increase was also reported in the study by Lee et al. [23]. In the present simulations, the decay of streamwise vorticity fluctuations was found to be more prominent for high Mach number flows.

It was observed that the variance of transverse vorticity fluctuations continuously decrease behind the shock. As opposed to the velocity fluctuations, vorticity fluctuations do return to isotropy behind the shock. An example is shown in Fig. 15 where transverse and streamwise vorticity fluctuations are seen to be converging to the same value downstream of the shock. However, the return to isotropy is delayed for the high Mach number flows as shown in Fig. 15. This was observed for all the computations undertaken. 


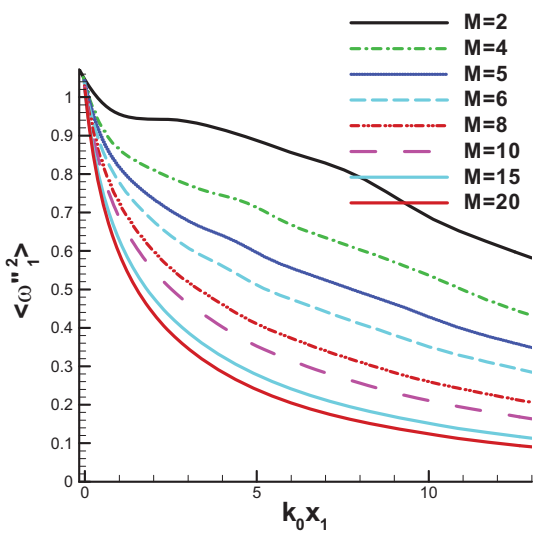

(a) $\operatorname{Re}_{\lambda}=6.8, M_{t}=0.121$

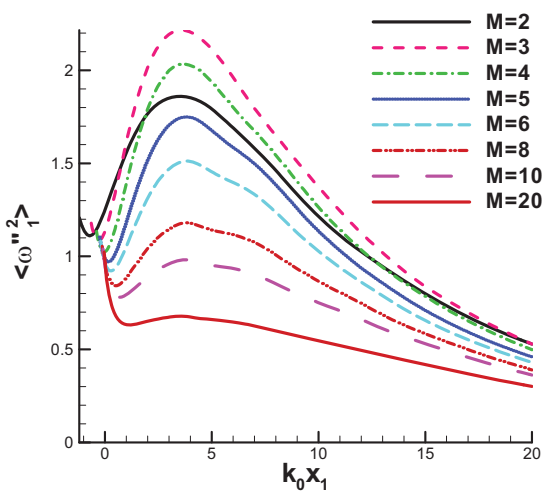

(c) $\operatorname{Re}_{\lambda}=22.4, M_{t}=0.275$

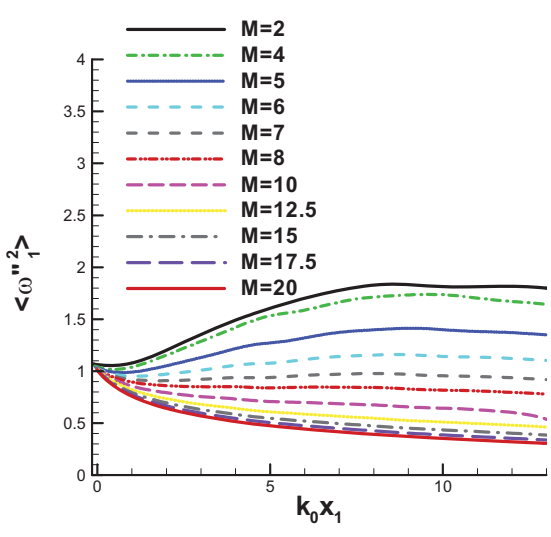

(b) $\operatorname{Re}_{\lambda}=15.8, M_{t}=0.126$

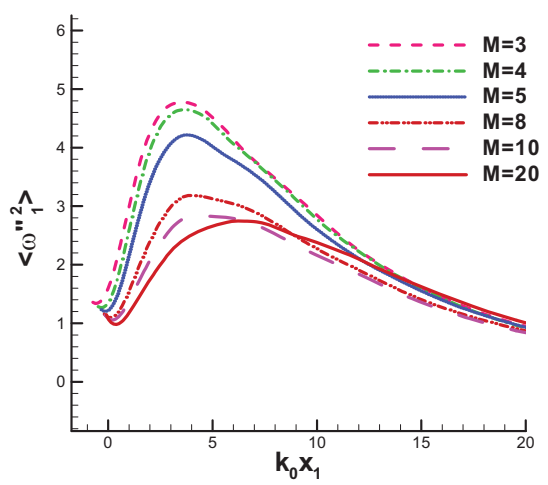

(d) $\operatorname{Re}_{\lambda}=39.7, M_{t}=0.376$

Fig. 14. Evolution of streamwise vorticity fluctuations variances behind the shock for a range of mean Mach number values.

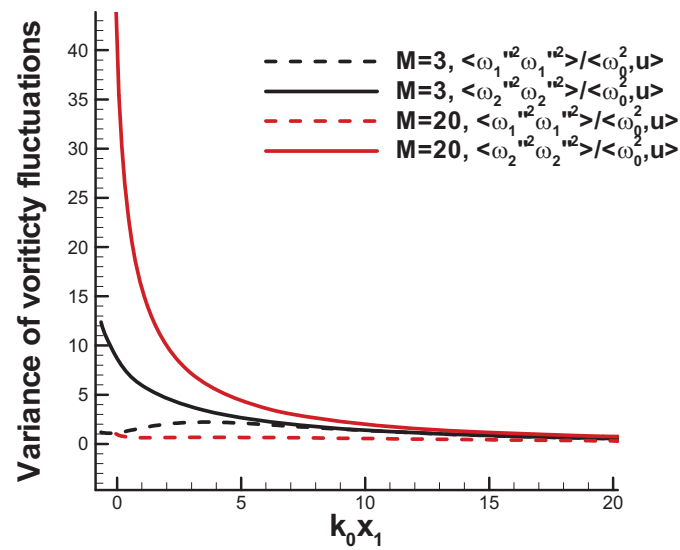

Fig. 15. Variation of amplification factor of transverse and streamwise vorticity fluctuations with two Mach number values for inflow of Case III $\left(\mathrm{Re}_{\lambda}=22.4, M_{t}=0.275\right)$. 


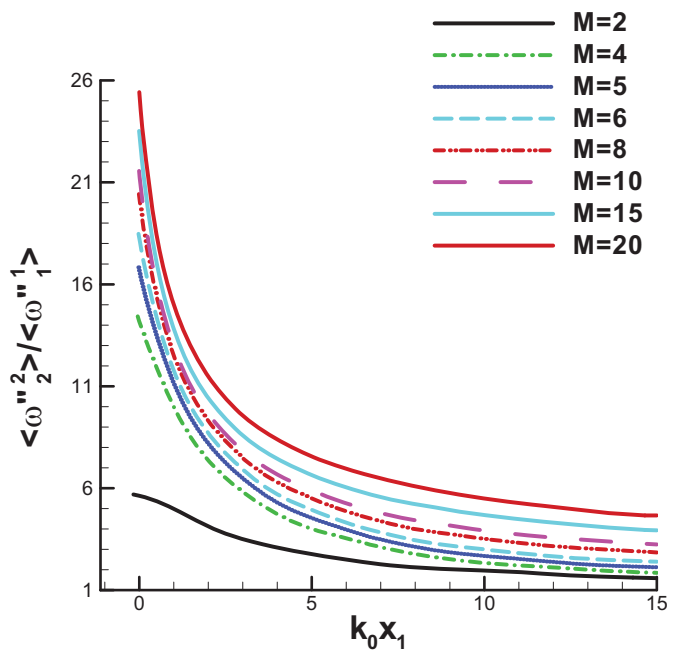

a) $\operatorname{Re}_{\lambda}=6.8, M_{t}=0.121$

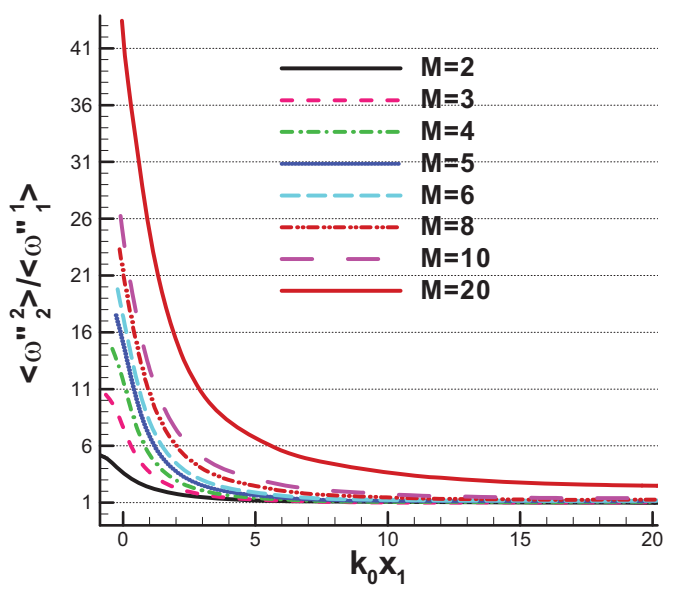

(c) $\operatorname{Re}_{\lambda}=22.4, M_{t}=0.275$

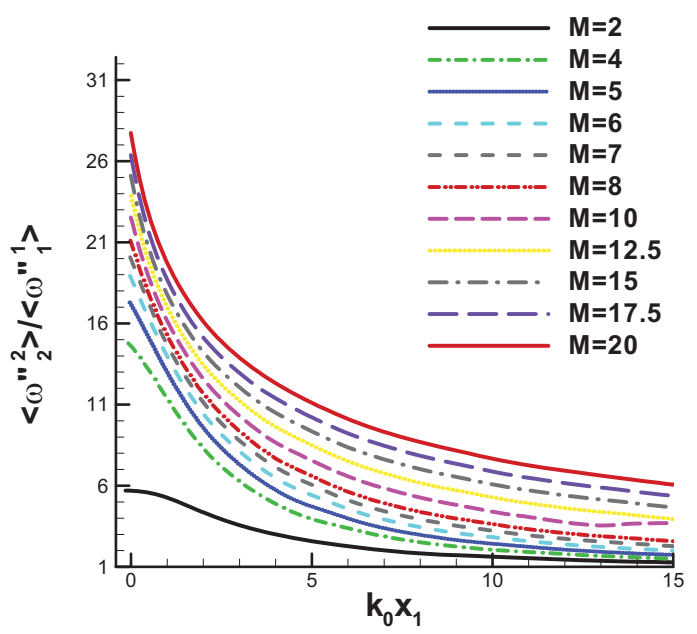

(b) $\operatorname{Re}_{\lambda}=15.8, M_{t}=0.126$

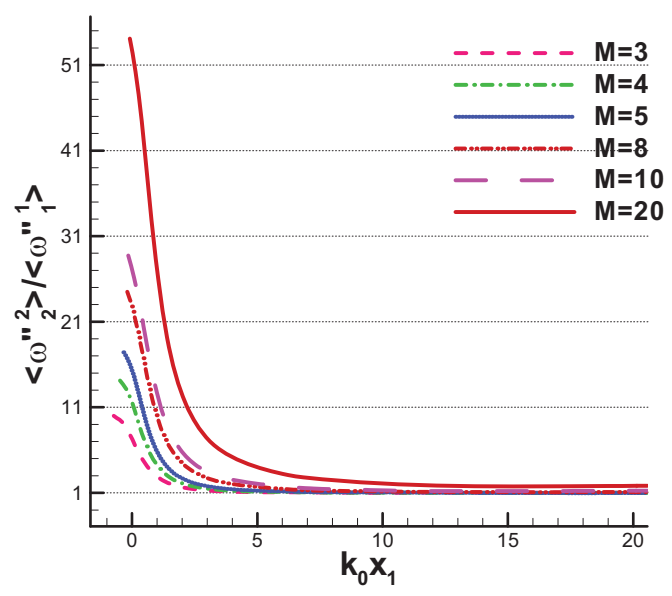

(d) $\operatorname{Re}_{\lambda}=39.7, M_{t}=0.376$

Fig. 16. Variation of amplification factor of transverse vorticity fluctuations in vorticity with change in Mach number of the incoming flow.

Similar to velocity fluctuations, we measure the anisotropy in the vorticity fluctuations by taking ratio of streamwise and transverse variances. These values are plotted in Fig. 16 for all the four cases considered here. It is again confirmed that for all the cases, return to isotropy is delayed for higher mean Mach number flows. Moreover, we also observed that as Reynolds number of the flow is increased the flow returns faster to the isotropy. These observations point to the fact that viscous dissipation of streamwise vorticity is the main cause of anisotropy of the vorticity fluctuations. This result can be very important for turbulence modeling of such flows. In real life and lab experiments the Reynolds number observed are much higher than those considered in this study and for purpose of developing models one can assume the vorticity fluctuations to be isotropic downstream of the shock. 
LIA also show increase in amplification of the transverse vorticity fluctuations with increase in Mach number. In Fig. 17, the amplification ratios predicted by LIA are compared against those obtained in the present study for low turbulence Mach numbers (Cases I and II). The shock fitting results were found to be matching the LIA prediction within 5\% for shocks as strong as Mach 10 flows. Previous studies in the field of shock turbulence interactions also showed good match with LIA for vorticity fluctuations but were never attempted with stronger than Mach 3 shocks.

Therefore, the suppression of vorticity tilting and stretching in post-shock flow strongly depends on the inflow conditions.

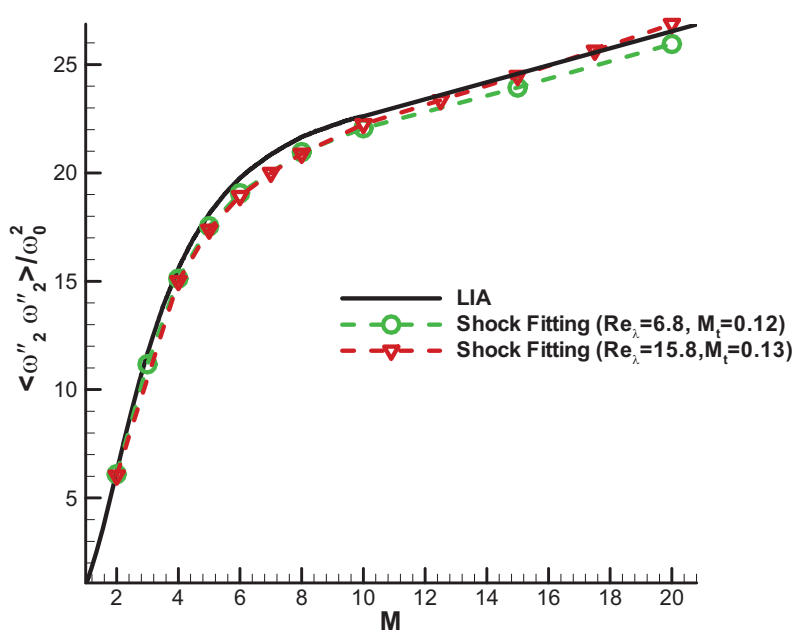

Fig. 17. Comparison of linear theory results with those obtained from shock-fitting results for small turbulence Mach numbers (Inflow of Cases I and II).

Evolutions of variance in streamwise vorticity fluctuations, $\widetilde{\omega_{1}^{\prime \prime} \omega_{1}^{\prime \prime}}$, is presented in Figs. 18 and 19 with the varying shock strengths but using same inflow turbulence of case VI and case VIII, respectively. Figure 18 shows that, for weaker than Mach 12 shocks in case VI, streamwise vorticity increases behind the shock. In case VIII, Figure 19 shows that, for weaker than Mach 7 shocks, streamwise vorticity increases behind the shock. Such increase is attributed to the non-linear tilting and stretching of vorticity and has also been reported in the past studies $[6,45]$. Both figures show that maximum values of variance of streamwise vorticity fluctuations first increase and then decrease as the shock strength is increased.

Furthermore, the peak of streamwise vorticity fluctuations is observed for shock and turbulence interactions with Mach 2.8 shock. In past, researchers [6, 45] considered weaker than Mach 3 shocks for such comparisons and concluded that effect of increasing shock strength is to simply increase the amplification of streamwise vorticity fluctuations. Although our results agree to these trend for lower Mach numbers, we see that for stronger than Mach 2.8 flows there is a decrease in streamwise vorticity. It is observed that non-linear tilting and stretching is suppressed by the viscous dissipation and streamwise vorticity continuously decreases downstream of the shock for stronger than Mach 12 shocks in Case VI and for stronger than Mach 7 shocks in Case VIII.

The results of case VII are quite similar to those of case VI. And the results of case IX are quite similar to those of case VIII. Because of the decrease of freestream isotropic turbulence from case VI to 
case IX, the variances in streamwise vorticity fluctuation of case VII and case IX are lower than those of case VI and case VIII, respectively.
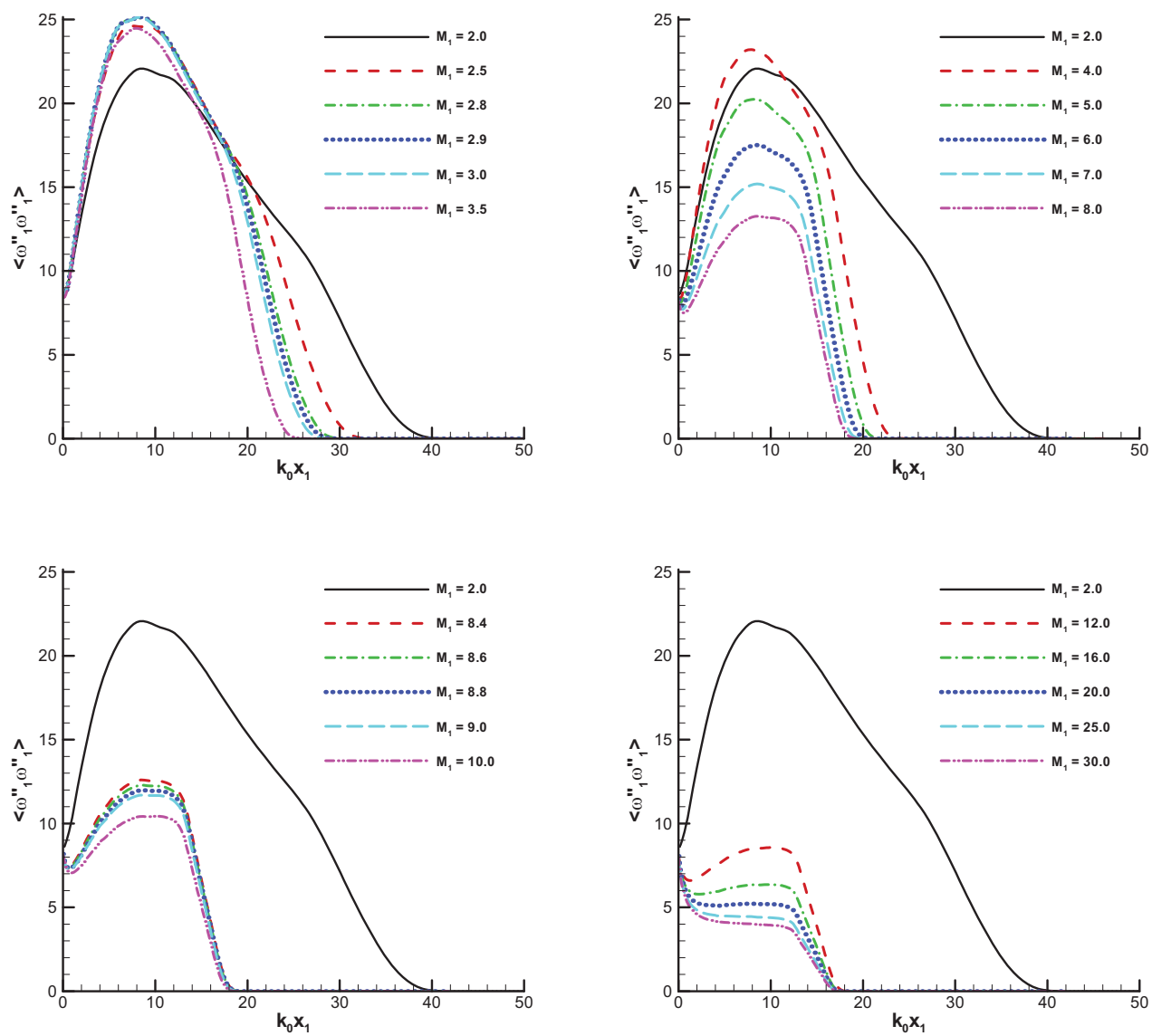

Fig. 18. Effect of increasing mean Mach number on the variance of streamwise vorticity for inflow conditions of case VI.

\subsection{Taylor Microscales}

In almost all of experimental studies, turbulent microscales show an overall increase of the microscales during the interaction with normal shock, which is in contradiction with theory and simulations [50]. Evolution of Taylor microscales $\lambda_{\alpha}$ from our shock fitting computation for Case I is presented in Fig. 20. All the microscales are normalized by the value of Taylor microscale, $\lambda_{0, u}$ immediately upstream of the shock in the isotropic turbulent flow. It can be observed in Fig. 20 that all the microscales decrease significantly as the flow passes through the shock. The streamwise microscale, $\lambda_{1}$ is much smaller than the transverse microscale, $\lambda_{2}$ immediately downstream of the shock. However, $\lambda_{1}$ rapidly evolves further downstream while increase in $\lambda_{2}$ is not much pronounced. Transverse microscale $\lambda_{2}$ remains less than the upstream value $\lambda_{0, u}$ in the computational domain for all the mean Mach numbers of incoming flow. 

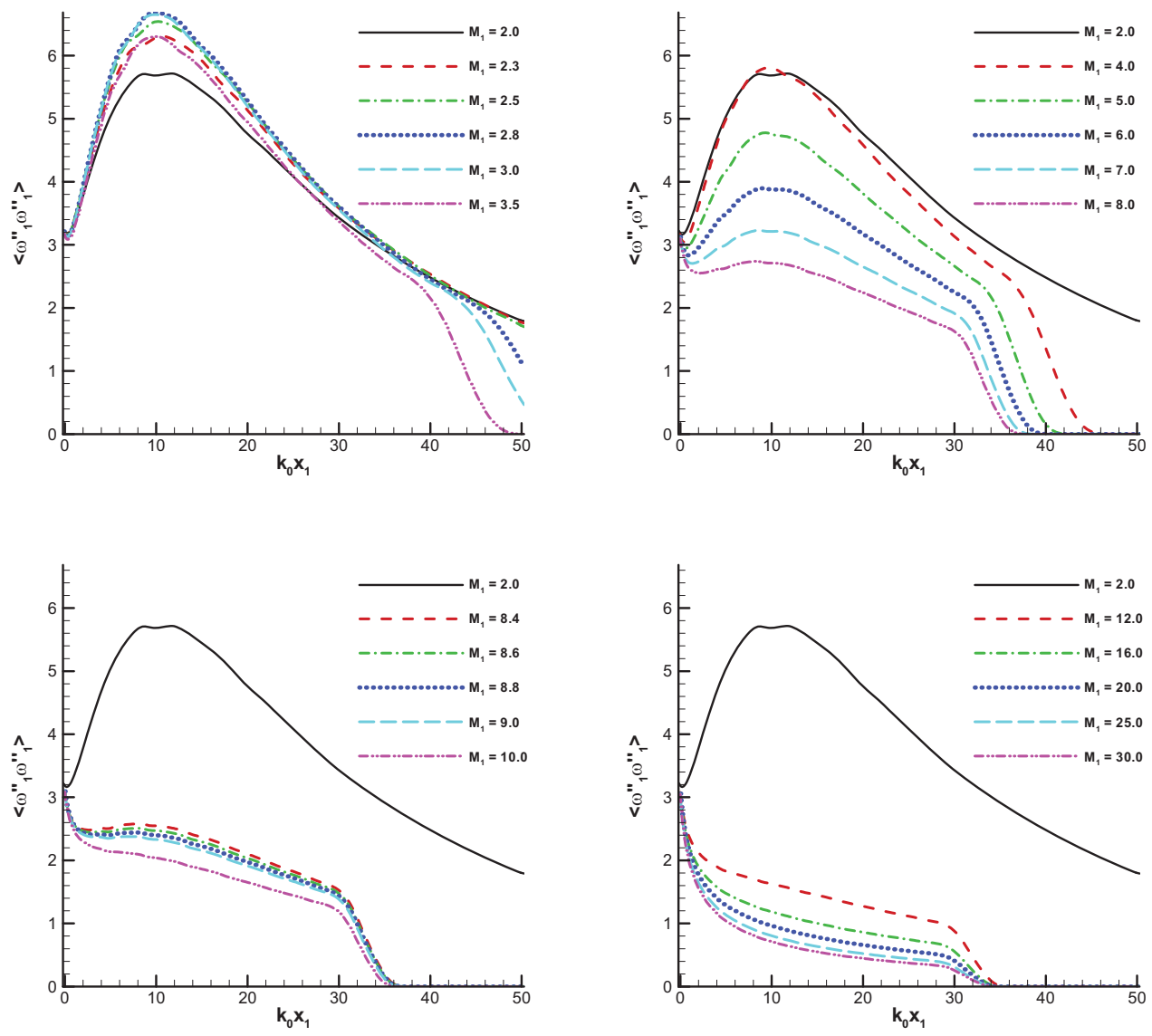

Fig. 19. Effect of increasing mean Mach number on the variance of streamwise vorticity for inflow conditions of case VIII.
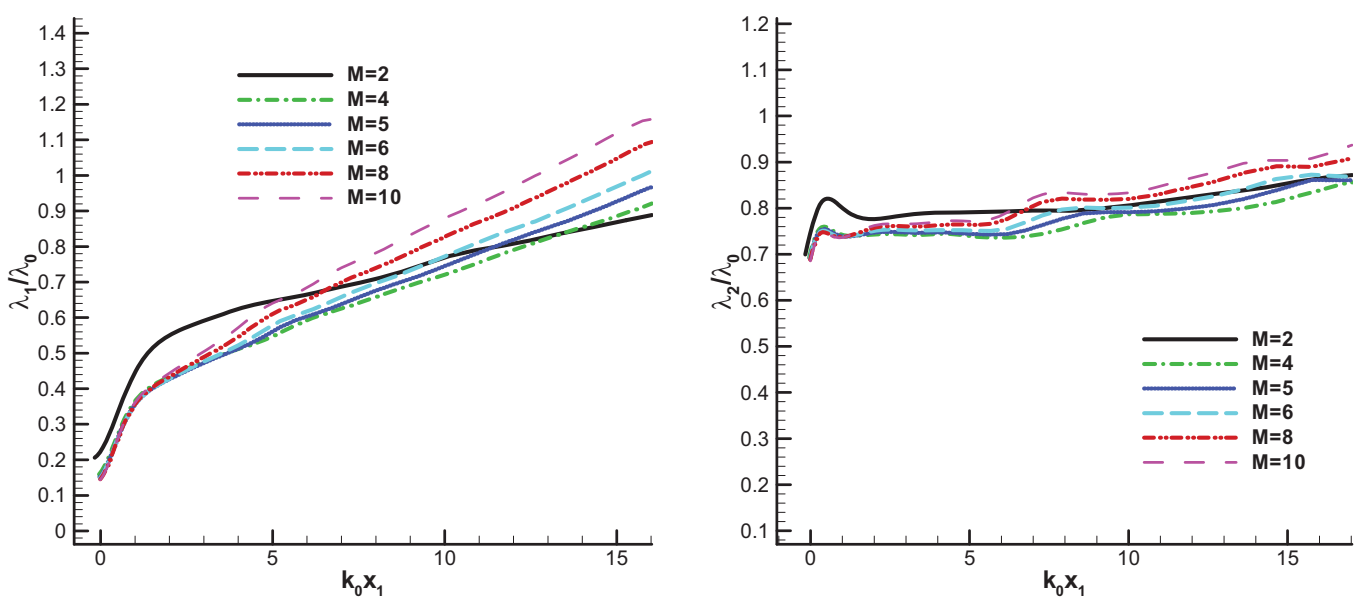

Fig. 20. Evolution of Taylor microscales corresponding to (a) streamwise and (b) transverse directions for a various values of Mach numbers. 
In all the cases with different shock strengths considered in this study, we found $\lambda_{1}$ to be greater than $\lambda_{2}$ beyond $k_{0} x_{1} \sim 10$. For the cases with higher Mach numbers of the incoming flow, all the microscales are smaller just behind the shocks but spatial evolution of $\lambda_{1}$ is much more rapid. Consequently, the point where $\lambda_{1}$ becomes larger than $\lambda_{2}$ was found to move closer to the shock as the Mach number of the incoming flow is increased while keeping $M_{t}$ and $\operatorname{Re}_{\lambda}$ fixed. Opposite to the computed results presented here, the linear theory predicts $\lambda_{2}>\lambda_{1}$ and the difference is expected to be larger for higher mean Mach number of the incoming flow. The discrepancy suggests fast viscous decay of streamwise derivative of velocity for high viscosity in case I.

For the weak shocks considered in the previous studies Lee et al [23] and Jamme et al. [24] showed excellent agreement with linear theory for computation of $\lambda_{2}$ values. These results are reproduced in the Fig. 21 and compared against those obtained by our shock fitting method. For this comparison we have chosen the $\lambda_{2}$ value immediately after the shock. Good agreement is observed between shock fitting and LIA results as the mean Mach number of incoming flow is increased.

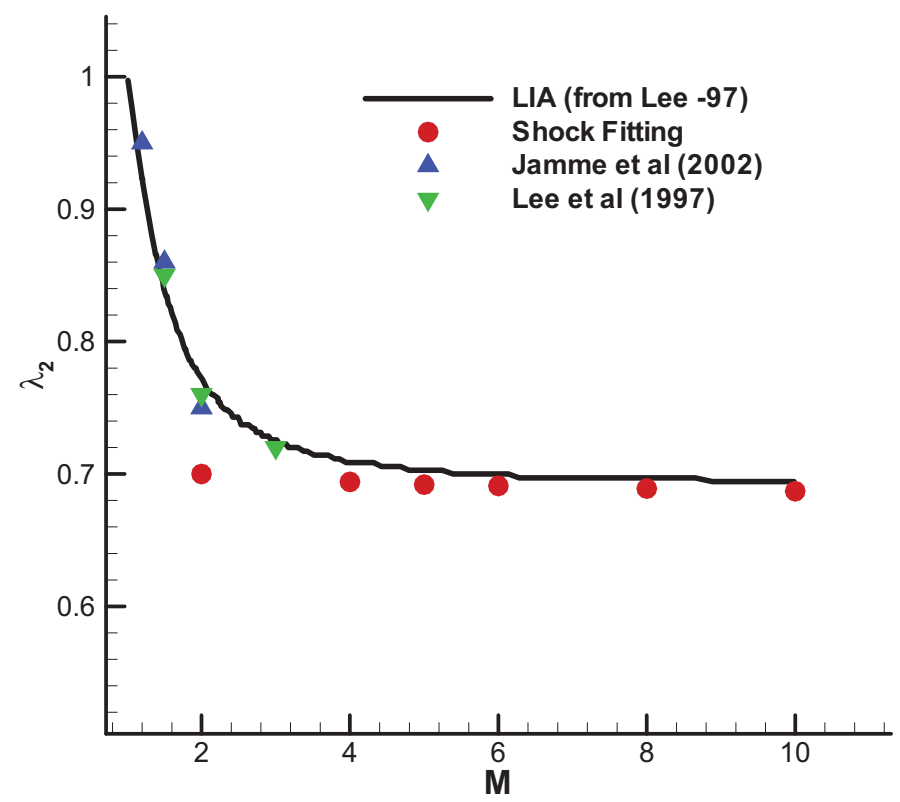

Fig. 21. Change in transverse Taylor microscale across the shock for various values of Mach numbers reported in literature.

\section{DEVELOPMENT AND VALIDATION OF HIGH-ORDER NON-EQUILIBRIUM SHOCK-FITTING CODE}

The SciDAC Science Application Partnerships (SAP), efficient low dissipation high order multiblock/embedded grid solver for shock turbulence interaction, is in support of the "Turbulence" Science Application project of Stanford University. The goal is to provide novel numerical methods and related tools to simulate complex turbulent flows with strong shocks in an efficient, stable, accurate, and reliable manner. 
Before shifting from SAP to SA, the main research achievements of UCLA research group on ADPDIS3D code, the software delivery of SAP, are as follows: 1. Finish implementing and validating the source terms of thermally non-equilibrium and chemically reactive flows. The ADPDIS3D code can be applied to the simulation of complex flows including thermal non-equilibrium and chemical reactions; 2 . Finish implementing backward Euler implicit method and semi-implicit Runge-Kutta (SIRK) implicit methods for high-order temporal integration. The schemes are implicit in chemical source terms. The backward Euler implicit method and SIRK schemes have been tested on one cell non-equilibrium simulation and the simulations of non-equilibrium and reactive flows.

We have implemented to current shock-fitting code the more realistic 11-species model of air with most recent thermo-chemical models of non-equilibrium air flow, including gas-mixture viscosity, heat conductivity, and diffusion coefficients [34,35], non-equilibrium reaction rates [36, 37], and equilibrium constants $[34,36,38]$. In the code, both the 22-reaction mechanism of LAURA and the 20-reaction mechanism of DPLR are included, with the detailed reactions given in Hash et al.'s paper [51]. It is assumed that translational and rotational energy modes are in equilibrium at the translation temperature (T), whereas vibration and electronic energy modes are in equilibrium at the vibration temperature (Tv). The non-equilibrium shock-fitting code can run cases with extremely high translational temperature. Currently, we are on the way finishing testing the 11-species shock-fitting code.

\subsection{Governing equations}

In current implementation, the gas consists of eleven species $(\mathrm{N} 2, \mathrm{O} 2, \mathrm{NO}, \mathrm{N}, \mathrm{O}, \mathrm{N} 2+, \mathrm{O} 2+, \mathrm{NO}+$, $\mathrm{N}+, \mathrm{O}+$, e). Governing equations consist of eleven mass conservation equations, three momentum conservation equations, and two energy conservation equations. Therefore, governing equations consists of sixteen equations, i.e.,

$$
\begin{gathered}
\frac{\partial \rho_{s}}{\partial t}+\frac{\partial}{\partial x_{j}}\left(\rho_{s} u_{j}\right)-\frac{\partial}{\partial x_{j}}\left(\rho D_{s} \frac{\partial y_{s}}{\partial x_{j}}\right)=\omega_{s} \\
\frac{\partial}{\partial t}\left(\rho u_{i}\right)+\frac{\partial}{\partial x_{j}}\left(\rho u_{i} u_{j}+p \delta_{i j}\right)-\frac{\partial}{\partial x_{j}}\left[\mu\left(\frac{\partial u_{i}}{\partial x_{j}}+\frac{\partial u_{j}}{\partial x_{i}}\right)-\frac{2}{3} \mu \frac{\partial u_{k}}{\partial x_{k}} \delta_{i j}\right]=0 \\
\frac{\partial \rho E}{\partial t}+\frac{\partial}{\partial x_{j}}\left(\rho H u_{j}\right)-\frac{\partial}{\partial x_{j}}\left[u_{i} \mu\left(\frac{\partial u_{i}}{\partial x_{j}}+\frac{\partial u_{j}}{\partial x_{i}}\right)-\frac{2}{3} u_{i} \mu \frac{\partial u_{k}}{\partial x_{k}} \delta_{i j}\right] \\
-\frac{\partial}{\partial x_{j}}\left(\rho \sum_{s=1}^{11} h_{s} D_{s} \frac{\partial y_{s}}{\partial x_{j}}\right)-\frac{\partial}{\partial x_{j}}\left(K \frac{\partial T}{\partial x_{j}}+K_{V} \frac{\partial T_{V}}{\partial x_{j}}\right)=0 \\
\frac{\partial \rho e_{V}}{\partial t}+\frac{\partial}{\partial x_{j}}\left(\rho e_{V} u_{j}\right)-\frac{\partial}{\partial x_{j}}\left(\rho \sum_{s=1}^{11} h_{V, s} D_{s} \frac{\partial y_{s}}{\partial x_{j}}\right)-\frac{\partial}{\partial x_{j}}\left(K_{V} \frac{\partial T_{V}}{\partial x_{j}}\right) \\
=\sum_{s=m o l} Q_{T-V, s}+\sum_{s=1}^{11} \omega_{s} e_{V, s}-p_{e} \frac{\partial u_{j}}{\partial x_{j}}-\sum_{s=6}^{10} \dot{n}_{e, s} \hat{I}_{s}+Q_{T-e}
\end{gathered}
$$

Where

$$
y_{s}=\frac{\left(\rho_{s} / M_{s}\right)}{\sum_{i=1}^{11}\left(\rho_{i} / M_{i}\right)} \quad \rho=\sum_{s=1}^{n} \rho_{s} \quad p=\sum_{s=1}^{11} p_{s}=\sum_{s=1}^{10} \frac{\rho_{s} \bar{R} T}{M_{s}}+\frac{\rho_{e} \bar{R} T_{V}}{M_{e}}
$$




$$
E=\frac{u_{i} u_{i}}{2}+\sum_{s=1}^{11} \frac{\rho_{s} e_{s}}{\rho} \quad H=E+\frac{p}{\rho} \quad h_{V, s}=\left\{\begin{array}{l}
e_{V, s} \quad(s \neq e) \\
e_{V, e}+\frac{\bar{R} T_{V}}{M_{e}}
\end{array}\right.
$$

\subsection{Energy relaxations}

In current model, there are two types of energy relaxations need to be considered. The translation -vibration energy relaxation is calculated as

$$
\begin{aligned}
& Q_{T-v, s}=\rho_{s} \frac{e_{v s}^{*}(T)-e_{v s}}{\tau_{v s}} \\
& \text { If T }<8,000 \mathrm{~K}, \tau_{v s}=\left\langle\tau_{s, L-T}\right\rangle=\frac{\sum_{r} y_{r}}{\sum_{r} y_{r} / \tau_{s r, L-T}} \\
& \text { If T }>8,000 \mathrm{~K}, \tau_{v s}=\left\langle\tau_{s, L-T}\right\rangle+\tau_{c s}=\frac{\sum_{r} y_{r}}{\sum_{r} y_{r} / \tau_{s r, L-T}}+\frac{1}{a_{s} \sigma_{v} N_{s}} \quad\left(a_{s}=\sqrt{\frac{8 \bar{R} T}{\pi M_{s}}}\right) \\
& \tau_{s r, L-T}=\frac{1}{p} \exp \left[A_{s r}\left(T^{-1 / 3}-0.015 \mu_{s r}^{1 / 4}\right)-18.42\right](\mathrm{p} \text { in atm }) \\
& A_{r}=1.16 \times 10^{-3} \mu_{s r}^{1 / 2} \theta_{v s}{ }^{4 / 3} \quad \mu_{s r}=M_{s} M_{r} /\left(M_{s}+M_{r}\right) \quad\left(\mathrm{M}_{s}\right. \text { in gram, not kilogram) } \\
& \sigma_{v}=10^{-21}(50,000 / T)^{2}
\end{aligned}
$$

The translation-electron energy relaxation is computed by

$$
Q_{T-e}=3 \bar{R} \rho_{e}\left(T-T_{V}\right) \sqrt{\frac{8 \bar{R} T_{V}}{\pi M_{e}}} \sum_{s=1}^{10} \frac{\rho_{r} \hat{N}}{M_{r}^{2}} \sigma_{e r}
$$

where the formula of the cross-section is,

$$
\sigma_{e r}=\left\{\begin{array}{lc}
\bar{a}_{r}+\bar{b}_{r} T_{V}+\bar{c}_{r} T_{V}^{2} & \text { (electron-neutral) } \\
\frac{8 \pi}{27} \frac{e^{4}}{k^{2} T_{V}^{2}} \ln \left(1+\frac{9 k^{3} T_{V}^{3}}{4 \pi N_{e} e^{6}}\right) & (\text { electron-ions })
\end{array}\right.
$$

\subsection{Chemical models}

The forward and backward reaction rate coefficients have the form of

$$
\begin{gathered}
k_{f}(\bar{T})=C_{f} \bar{T}^{\eta_{f}} \exp \left(-\theta_{f} / \bar{T}\right) \\
k_{b}(T)=\frac{k_{f}(T)}{k_{\text {eq }}(T)}
\end{gathered}
$$

For dissociation reactions, $\bar{T}=\sqrt{T T_{V}}$. For electron impact ionization reactions, the control temperature is $\mathrm{T}_{\mathrm{V}}$. For the other reactions, the control temperature is $\mathrm{T}$. 
The equilibrium constant is calculated either from curve fits $[34,36]$ or from the free Gibbs energy [38],

$$
\ln k_{e q}=-\frac{\Delta_{f} G^{0}}{\bar{R} T}
$$

where $\Delta_{f} G^{0}$ is the reaction standard Gibbs energy, which is the sum of the standard Gibbs energies of the reaction products minus the sum of standard Gibbs energies of reactants,

$$
\ln k_{\text {eq }}=\sum_{s=\text { reactant }}\left(\frac{h_{s}}{R T}-\frac{S_{s}}{R}\right)-\sum_{s=\text { product }}\left(\frac{h_{s}}{R T}-\frac{S_{s}}{R}\right)
$$

\begin{tabular}{|c|c|c|c|c|}
\hline Index & Reaction & $\mathrm{C}_{\mathrm{f}}\left(\mathrm{m}^{3} / \mathrm{kmol}-\mathrm{s}\right)$ & $\eta_{\mathrm{f}}$ & $\theta_{\mathrm{f}}(\mathrm{K})$ \\
\hline 1 & $\begin{array}{c}\mathrm{N}_{2}+\mathrm{M} \leftrightarrow \mathrm{N}+\mathrm{N}+\mathrm{M}\left(\mathrm{M}=\mathrm{N}_{2}, \mathrm{O}_{2}, \mathrm{NO}\right) \\
\mathrm{N}_{2}+\mathrm{M} \leftrightarrow \mathrm{N}+\mathrm{N}+\mathrm{M}(\mathrm{M}=\mathbf{N}, \mathbf{O})\end{array}$ & $\begin{array}{l}7.0 \mathrm{D}+18 \\
3.0 \mathrm{D}+19 \\
\end{array}$ & $\begin{array}{r}-1.60 \\
-1.60 \\
\end{array}$ & $\begin{array}{l}113200 \\
113200 \\
\end{array}$ \\
\hline 2 & $\begin{array}{c}\mathrm{O}_{2}+\mathrm{M} \leftrightarrow \mathrm{O}+\mathrm{O}+\mathrm{M}\left(\mathrm{M}=\mathrm{N}_{2}, \mathrm{O}_{2}, \mathrm{NO}\right) \\
\mathrm{O}_{2}+\mathrm{M} \leftrightarrow \mathrm{O}+\mathrm{O}+\mathrm{M}(\mathrm{M}=\mathrm{N}, \mathrm{O})\end{array}$ & $\begin{array}{l}2.0 \mathrm{D}+18 \\
1.0 \mathrm{D}+19\end{array}$ & $\begin{array}{r}-1.50 \\
-1.50\end{array}$ & $\begin{array}{l}59360 \\
59360\end{array}$ \\
\hline 3 & $\begin{array}{c}\mathrm{NO}+\mathrm{M} \leftrightarrow \mathrm{N}+\mathrm{O}+\mathrm{M}\left(\mathrm{M}=\mathrm{N}_{2}, \mathrm{O}_{2}, \mathrm{NO}\right) \\
\mathrm{NO}+\mathrm{M} \leftrightarrow \mathrm{N}+\mathrm{O}+\mathrm{M}(\mathrm{M}=\mathrm{N}, \mathrm{O})\end{array}$ & $\begin{array}{l}5.2 \mathrm{D}+12 \\
1.1 \mathrm{D}+14\end{array}$ & $\begin{array}{l}0.00 \\
0.00\end{array}$ & $\begin{array}{l}75500 \\
75500\end{array}$ \\
\hline 4 & $\mathrm{~N}_{2}+\mathrm{O} \leftrightarrow \mathrm{NO}+\mathrm{N}$ & $5.7 \mathrm{D}+9$ & 0.42 & 42938 \\
\hline 5 & $\mathrm{NO}+\mathrm{O} \leftrightarrow \mathrm{O}_{2}+\mathrm{N}$ & $8.4 \mathrm{D}+9$ & 0.00 & 19400 \\
\hline 6 & $\mathbf{N}_{2}+\mathbf{e} \leftrightarrow \mathrm{N}+\mathrm{N}+\mathrm{e}$ & $3.0 \mathrm{D}+21$ & -1.60 & 113200 \\
\hline 7 & $\mathbf{N}+\mathbf{e} \leftrightarrow \mathbf{N}^{+}+\mathbf{e}+\mathbf{e}$ & $2.5 \mathrm{D}+31$ & -3.82 & 168600 \\
\hline 8 & $\mathbf{O}+\mathbf{e} \leftrightarrow \mathbf{O}^{+}+\mathrm{e}+\mathrm{e}$ & $3.9 \mathrm{D}+30$ & -3.78 & 158500 \\
\hline 9 & $\mathbf{N}+\mathbf{O} \leftrightarrow \mathrm{NO}^{+}+\mathbf{e}$ & $5.3 \mathrm{D}+9$ & 0.00 & 31900 \\
\hline 10 & $\mathbf{N}+\mathbf{N} \leftrightarrow \mathbf{N}_{2}^{+}+\mathbf{e}$ & $4.4 \mathrm{D}+4$ & 1.50 & 67500 \\
\hline 11 & $\mathbf{O}+\mathbf{O} \leftrightarrow \mathbf{O}_{2}^{+}+\mathrm{e}$ & 7.1D-1 & 2.70 & 80600 \\
\hline 12 & $\mathrm{O}^{+}+\mathrm{N}_{2} \leftrightarrow \mathbf{N}_{2}^{+}+\mathbf{O}$ & $9.1 \mathrm{D}+8$ & 0.36 & 22800 \\
\hline 13 & $\mathrm{O}^{+}+\mathrm{NO} \leftrightarrow \mathrm{N}^{+}+\mathrm{O}_{2}$ & $1.4 \mathrm{D}+2$ & 1.90 & 26600 \\
\hline 14 & $\mathrm{NO}^{+}+\mathrm{O}_{2} \leftrightarrow \mathrm{O}_{2}^{+}+\mathrm{NO}$ & $2.4 \mathrm{D}+10$ & 0.41 & 32600 \\
\hline 15 & $\mathrm{NO}^{+}+\mathrm{N} \leftrightarrow \mathrm{N}_{2}{ }^{+}+\mathrm{O}$ & $7.2 \mathrm{D}+10$ & $\mathbf{0 . 0 0}$ & 35500 \\
\hline 16 & $\mathrm{NO}^{+}+\mathrm{O} \leftrightarrow \mathrm{N}^{+}+\mathrm{O}_{2}$ & $1.0 \mathrm{D}+9$ & 0.50 & 77200 \\
\hline 17 & $\mathrm{O}_{2}^{+}+\mathrm{N} \leftrightarrow \mathrm{N}^{+}+\mathrm{O}_{2}$ & $8.7 \mathrm{D}+10$ & 0.14 & 28600 \\
\hline 18 & $\mathrm{O}_{2}^{+}+\mathrm{N}_{2} \leftrightarrow \mathrm{N}_{2}^{+}+\mathrm{O}_{2}$ & $9.9 \mathrm{D}+9$ & 0.00 & 40700 \\
\hline 19 & $\mathrm{NO}^{+}+\mathrm{N} \leftrightarrow \mathrm{O}^{+}+\mathrm{N}_{2}$ & $3.4 \mathrm{D}+10$ & -1.08 & 12800 \\
\hline 20 & $\mathrm{NO}^{+}+\mathrm{O} \leftrightarrow \mathrm{O}_{2}^{+}+\mathrm{N}$ & $7.2 \mathrm{D}+9$ & 0.29 & 48600 \\
\hline 21 & $\mathrm{O}_{2}^{+}+\mathrm{O} \leftrightarrow \mathrm{O}^{+}+\mathrm{O}_{2}$ & $4.0 \mathrm{D}+9$ & -0.09 & 18000 \\
\hline 22 & $\mathbf{N}^{+}+\mathbf{N}_{2} \leftrightarrow \mathbf{N}_{2}^{+}+\mathbf{N}$ & $1.0 \mathrm{D}+9$ & 0.50 & 12200 \\
\hline
\end{tabular}

Table 2. Chemical reactions for the 11-species air

\subsection{Transportation coefficients}

The following section lists the formula used to compute transportation coefficients. Due to the complexity of the 11-species air model, the calculations are not straightforward. 
Viscosity of air mixture

$$
\mu=\sum_{S \neq e} \frac{m_{S} \gamma_{S}}{\sum_{r \neq e} \gamma_{r} \Delta_{S r}^{(2)}(T)+\gamma_{e} \Delta_{S e}^{(2)}\left(T_{V}\right)}+\frac{m_{e} \gamma_{e}}{\sum \gamma_{r} \Delta_{e r}^{(2)}\left(T_{V}\right)}(\mathrm{g} / \mathrm{cm}-\mathrm{sec})
$$

Translational thermal conductivity of heavy particles

$$
\begin{gathered}
K_{T}=\frac{15}{4} k \sum_{S \neq e} \frac{\gamma_{S}}{\sum_{r \neq e} a_{S r} \gamma_{r} \Delta_{S r}^{(2)}(T)+3.54 \gamma_{e} \Delta_{S e}^{(2)}\left(T_{V}\right)}(\mathrm{J} / \mathrm{cm}-\mathrm{sec}-\mathrm{K}) \\
a_{S r}=1+\frac{\left[1-\left(m_{S} / m_{r}\right)\right]\left[0.45-2.54\left(m_{S} / m_{r}\right)\right]}{\left[1+\left(m_{S} / m_{r}\right)\right]^{2}}
\end{gathered}
$$

Rotational thermal conductivity of heavy particles (Fully excited)

$$
K_{R}=k \sum_{s=m o l} \frac{\gamma_{s}}{\sum_{r \neq e} \gamma_{r} \Delta_{S r}^{(1)}(T)+\gamma_{e} \Delta_{S e}^{(1)}\left(T_{V}\right)}(\mathrm{J} / \mathrm{cm}-\mathrm{sec}-\mathrm{K})
$$

Vibrational-electronic thermal conductivity of heavy particles

$$
K_{V}=k \sum_{s=1}^{10} \frac{C_{V_{S}, V^{\gamma_{S}} / R}}{\sum_{r \neq e} \gamma_{r} \Delta_{S r}^{(1)}(T)+\gamma_{e} \Delta_{S e}^{(1)}\left(T_{V}\right)}(\mathrm{J} / \mathrm{cm}-\mathrm{sec}-\mathrm{K})
$$

Electron thermal conductivity

$$
K_{e}=\frac{15}{4} k \frac{\gamma_{e}}{\sum_{r \neq e} 1.45 \gamma_{r} \Delta_{e r}^{(2)}\left(T_{V}\right)+\gamma_{e} \Delta_{e e}^{(2)}\left(T_{V}\right)}(\mathrm{J} / \mathrm{cm}-\mathrm{sec}-\mathrm{K})
$$

Collision terms

$$
\begin{gathered}
\Delta_{S r}^{(1)}(T)=\frac{8}{3}\left[\frac{2 m_{S} m_{r}}{\pi R T\left(m_{s}+m_{r}\right)}\right]^{1 / 2} 10^{-20} \pi \Omega_{S r}^{(1,1)}(T)(\mathrm{cm}-\mathrm{sec}) \\
\Delta_{S r}^{(2)}(T)=\frac{16}{3}\left[\frac{2 m_{S} m_{r}}{\pi R T\left(m_{s}+m_{r}\right)}\right]^{1 / 2} 10^{-20} \pi \Omega_{S r}^{(2,2)}(T)(\mathrm{cm}-\mathrm{sec})
\end{gathered}
$$

Collision integrals involving neutrals (Non-Coulombic collision integrals)

$$
\pi \Omega_{S r}^{(l, j)}(T)=D T^{\left[A(\ln T)^{2}+B \ln T+C\right]}\left(\begin{array}{c}
0 \\
\left(\mathrm{~A}^{2}\right)
\end{array}\right.
$$

Coulombic collision integrals for electron-ion, ion-ion, and electron-electron collision 


$$
\pi \Omega^{(l, l)}(T)=5.0 \times 10^{15} \pi\left(\lambda_{D} / T^{*}\right)^{2} \ln \left\{D_{l} T^{*}\left[1-C_{l} \exp \left(-c_{l} T^{*}\right)\right]+1\right\}\left(\mathrm{A}^{2}\right)
$$

where

$$
\begin{gathered}
T^{*}=\frac{\lambda_{D}}{e^{2} /(k T)} \\
\lambda_{D}=\sqrt{\frac{k T}{4 \pi N_{e} e^{2}}}
\end{gathered}
$$

Species diffusion coefficients

$$
D_{s}=\frac{\left(1-y_{s}\right)}{\sum_{r \neq s}\left(y_{r} / D_{s r}\right)}
$$

Where $\mathrm{y}_{\mathrm{s}}$ is the molar fraction. For binary diffusion between heavy particles,

$$
D_{s r}=\frac{k T}{p \Delta_{s r}^{(1)}(T)}
$$

For electrons,

$$
D_{e r}=\frac{k T_{V}}{p \Delta_{e r}^{(1)}\left(T_{V}\right)}
$$

For ambipolar diffusion (no charge separation),

$$
\begin{gathered}
D_{\text {ion }}^{a}=2 D_{\text {ion }} \\
D_{e}=M_{e} \frac{\sum_{s=6}^{10} D_{s}^{a} \gamma_{s}}{\sum_{s=6}^{10} M_{s} \gamma_{s}}
\end{gathered}
$$

\subsection{Results of code test}

To validate the newly implemented non-equilibrium shock-fitting code, we have run test cases of hypersonic flows. Currently, we are on the way finishing testing the code. However, final results are only available for the test case relating to Lobb's 1964 experiment. It is discussed in this section.

\subsubsection{A Mach 15 flow over a sphere}

Flow condition for the Mach 15 flow are listed as follows,

Nose diameter of sphere $=0.5$ in

$\mathrm{M}_{\infty}=15.3$

$\operatorname{Re}=26480$

$\mathrm{T}_{\infty}^{\infty}=293 \mathrm{~K}$

$\rho_{\infty}=7.83 \mathrm{E}-3 \mathrm{~kg} / \mathrm{m}^{3}$

Mass fractions in free stream

$$
\begin{aligned}
& \mathrm{N}_{2}=0.79 \\
& \mathrm{O}_{2}=0.21
\end{aligned}
$$




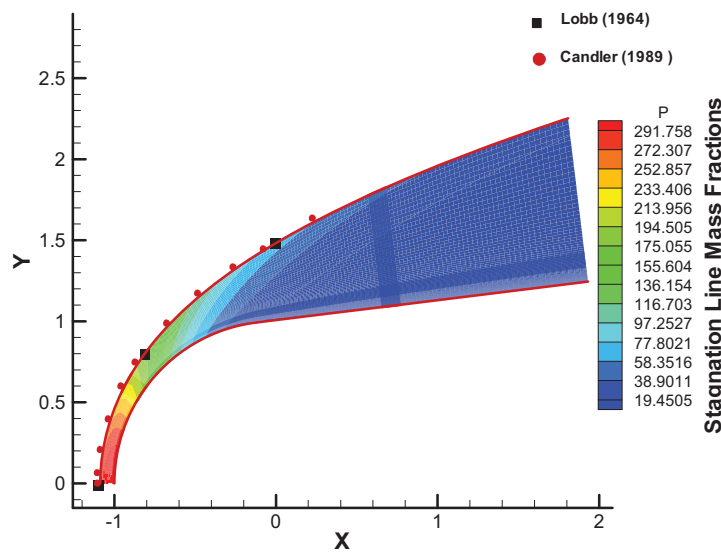

Fig. 22. Shock standoff distance, Pressure contours.

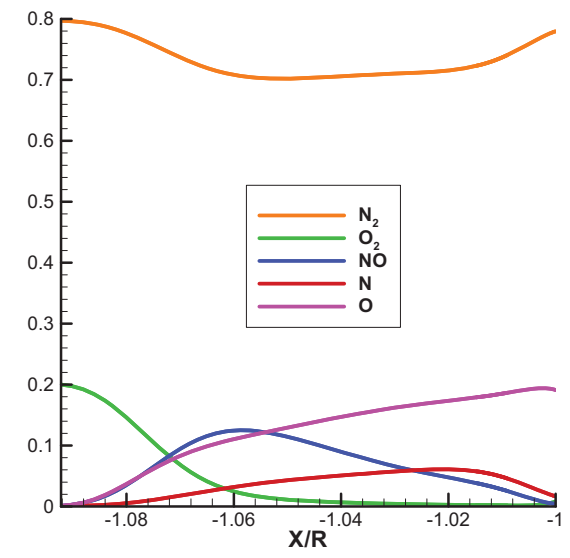

Fig. 23. Species concentration along stagnation line.

This test case has a Mach number high enough for thermal and chemical non equilibrium to exist. We get very good agreement on the shock standoff distance with Lobb's experimental results and Candlers's simulations as shown in Fig. 22. The shock standoff distance matches exactly at the stagnation point and away from it. Also shown in the figure are the pressure contours. Figure 23 shows species mass fractions at stagnation line. Immediately behind the shock the temperature rises to about $12000 \mathrm{~K}$. We assume that there is no vibration relaxation across the shock so vibration temperature is still at its free stream value. Due to the high temperature the air starts reacting and energy relaxation becomes important. There is a region of nonequilibrium flow behind the shock. Current simulations are at constant wall temperature of $1000 \mathrm{~K}$. In , we see that Oxygen almost disappears after some distance and converts to mono atomic oxygen. The species, NO, reaches maximum somewhere in the middle of the stagnation region. Figure 24 shows relaxation of vibration and translation temperatures. There is thermal nonequilibrium for most of the flow field except near the surface where they both are equal.

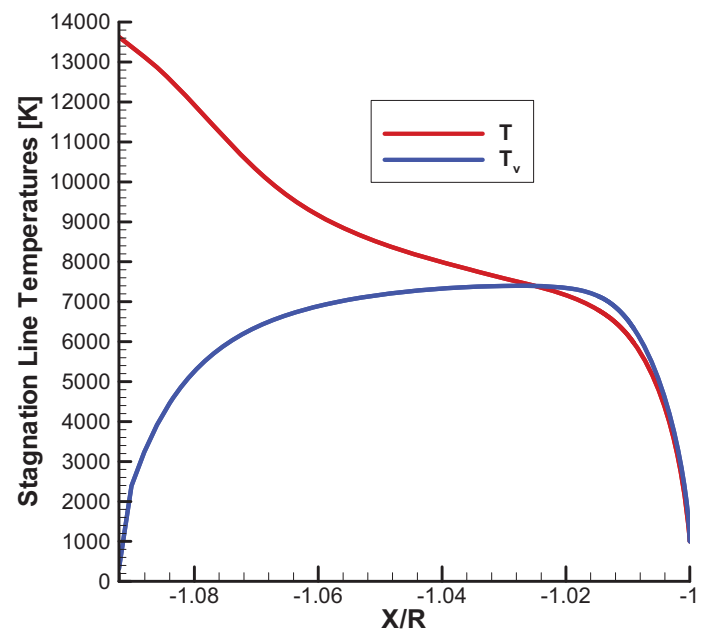

Fig. 24. Variation of temperature along stagnation line. 


\subsubsection{Gnoffo's Air Dissociation over 1 Meter Radius Cylinder}

Figure 25 shows the mesh and flow conditions of the test case: 5 -species air over a 1-meter radius cylinder. The temperatures on the cylinder are equal to Tw $(=500 \mathrm{~K})$. Catalytic boundary conditions are applied on the wall for species mass fraction. Total density is computed from pressure and translational temperature. Then species densities are calculated with total density and mass fraction. Total energy and vibration energy are calculated using species densities and two temperatures. The mass fractions of initial gas are as follows,

$$
\mathrm{C}_{\mathrm{N} 2}=0.76 \quad \mathrm{C}_{\mathrm{O} 2}=0.24 \quad \mathrm{C}_{\mathrm{NO}}=\mathrm{C}_{\mathrm{N}}=\mathrm{C}_{\mathrm{O}}=0
$$

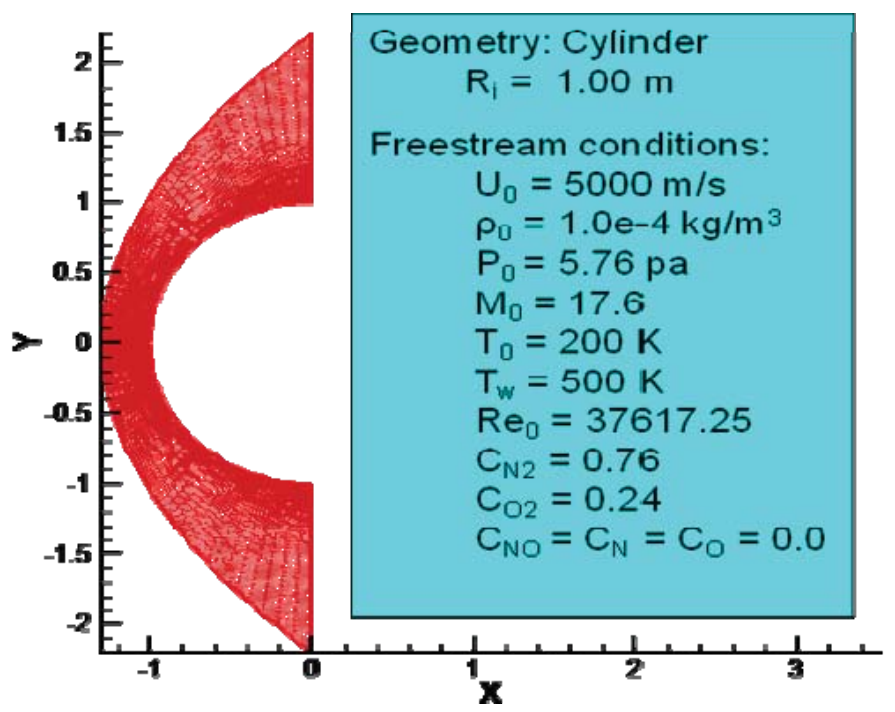

Fig. 25. Mesh sturcture and flow conditions of the test case.

To make the results comparable, flow conditions are exactly the same as what Gnoffo used in his simulation. The simulation results are compared with Gnoffo's results obtained from Laura. Figure 26 compares flow field contours obtained from current shock-fitting code with those obtained from Laura code. From the contours of pressure, temperatures, and NO density, it is found that shock standoff distances of the two sets of simulations have a good agreement. In addition, the flow fields near the wall have a good agreement. Near the shock, there is small discrepancy between the two sets of solution, mainly due to the different treatment of shock wave. Unlike the shock-fitting code, shock-capturing TVD scheme is applied in Laura code. Figure 26(c) shows that the vibration temperature of shock-fitting solution is significant different from that of Laura in the shock layer, which is mainly caused by the different models of vibration and electronic energy. Laura code used curved fitted vibration and electronic energy [52], whereas we used separate models for vibration energy and electronic energy. 


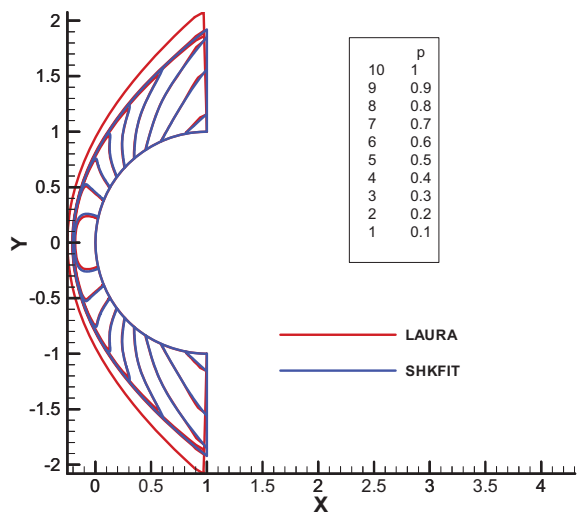

(a) Pressure

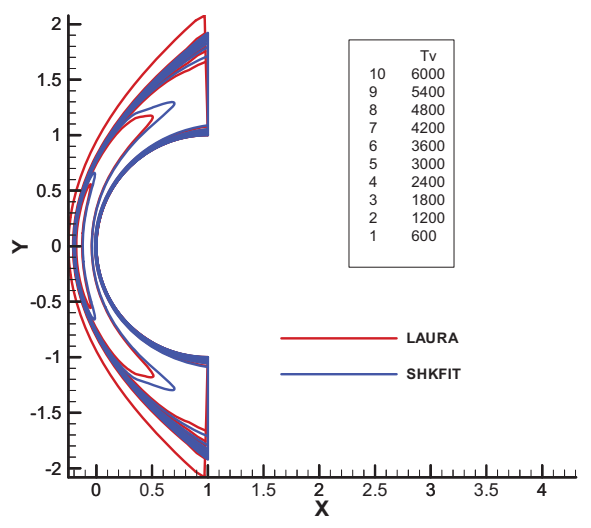

(c) Vibration temperature

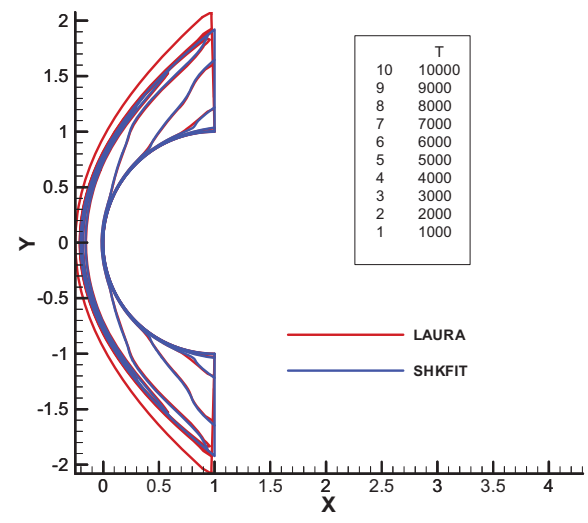

(b) Translation temperature

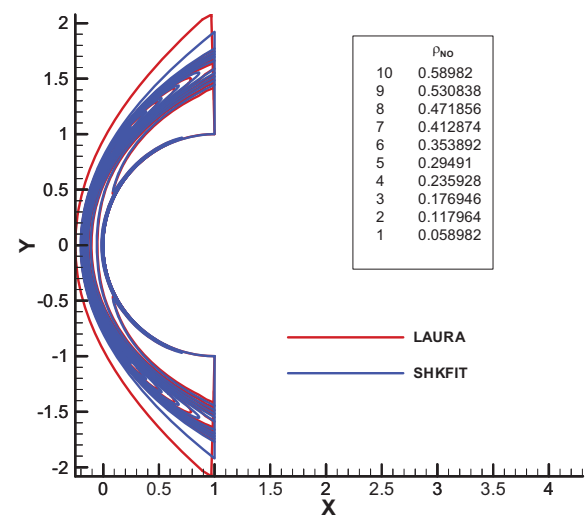

(d) Species density of $\mathrm{NO}$

Fig. 26. Comparisons of flow field contours obtained from shock-fitting code with those obtained from Laura simulation.

Since we have detailed flow field information obtained from the Laura code, we can also compare the distributions of flow variables along the stagnation line or along the cylinder surface. For example, figure 27 compares flow variables along the stagnation line obtained from current shock-fitting code with those obtained from Laura code. These two figures also show that shock standoff distances of the two sets of simulations have a good agreement considering the different treatment of the bow shock. The distributions of temperatures and species densities along the stagnation line have a good agreement near the wall and have small discrepancy near the shock. Again, the discrepancy near the shock is due to the different treatment of shock wave. Overall, Figures 26 and 27 indicate that our shock-fitting nonequilibrium flow solver is reliable for the simulation of strong shock and turbulence interaction. 


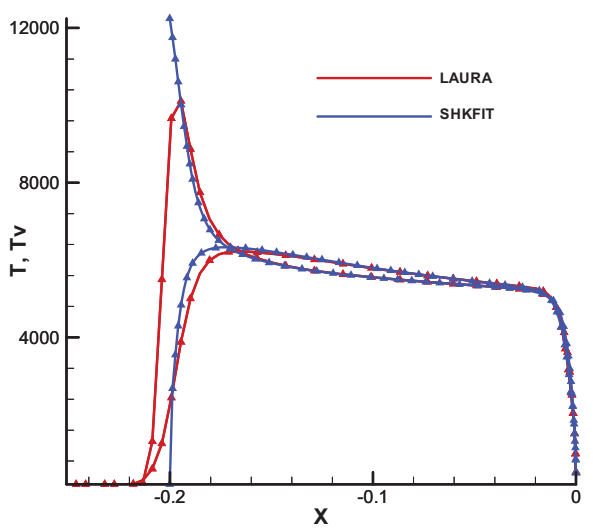

(a) Temperatures

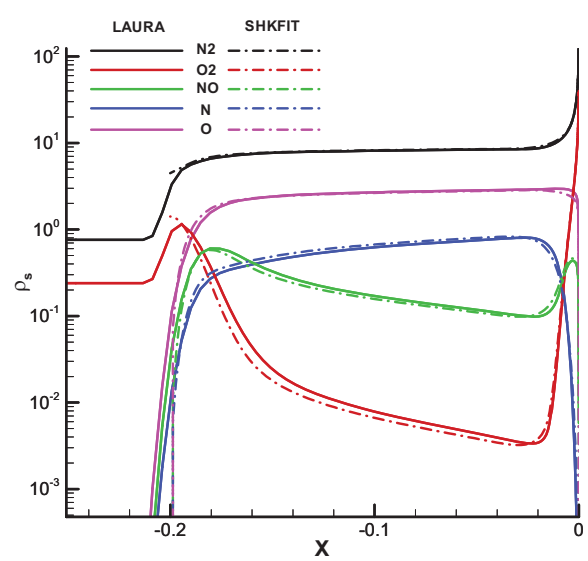

(b) Species densities

Fig. 27. Comparisons of flow variables along the stagnation line obtained from shock-fitting code with those obtained from Laura simulation.

\subsubsection{A Mach 10 Flat-Plate Boundary Layer with Thermal Equilibrium}

The test case is got from Hudson's thesis [53]. The flow conditions of the flat-plate boundary layer are as follows,

$$
\begin{aligned}
& M=10 \quad T_{\infty}=278 \mathrm{~K} \quad p_{\infty}=0.045 \mathrm{~atm} \quad u_{\infty}=3351 \mathrm{~m} / \mathrm{s} \\
& \rho_{\infty}=0.0568 \mathrm{~kg} / \mathrm{m}^{3} \quad \operatorname{Re}_{\infty}=9.8425 \times 10^{6} / \mathrm{m} \\
& \mathrm{C}_{\mathrm{N} 2}=0.78 \quad \mathrm{C}_{\mathrm{O} 2}=0.22 \quad \mathrm{C}_{\mathrm{NO}}=\mathrm{C}_{\mathrm{N}}=\mathrm{C}_{\mathrm{O}}=0
\end{aligned}
$$

Our numerical results are compared with Hudson's theoretical. Specifically, the temperature and velocity profiles across the boundary layer at $\mathrm{x}=0.4 \mathrm{~m}$ are compared.

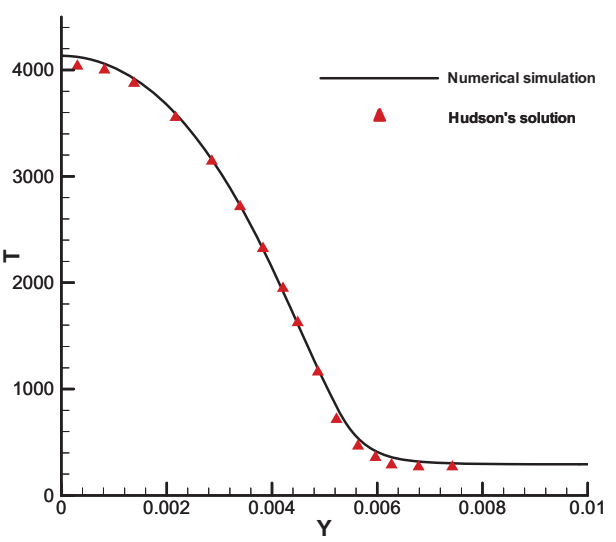

(a) temperature profile

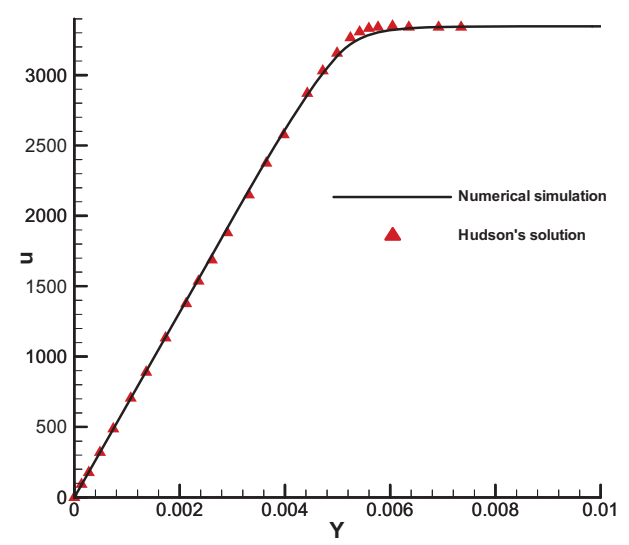

(b) streamwise velocity profile

Fig. 28. Comparisons of boundary layer profiles. 
Figure 28 shows that the boundary-layer profiles obtained from our simulation have agreement with the theoretical solutions of Hudson. The results indicate that our shock-fitting non-equilibrium flow solver is reliable.

\subsubsection{Nitrogen dissociation over 1 inch radius cylinder}

The flow conditions of Hornung's experiment [54] are listed in Fig. 29, together with a schematic of the grid used in numerical simulations. This experimental study focused on the flow field relating to Nitrogen dissociation over 1 inch radius cylinder. The mass fractions of initial gas are as follows,

$$
\begin{aligned}
& \mathrm{C}_{\mathrm{N} 2}=0.927, \mathrm{C}_{\mathrm{N}}=0.073 \\
& \mathrm{C}_{\mathrm{O} 2}=\mathrm{C}_{\mathrm{NO}}=\mathrm{C}_{\mathrm{O}}=0
\end{aligned}
$$

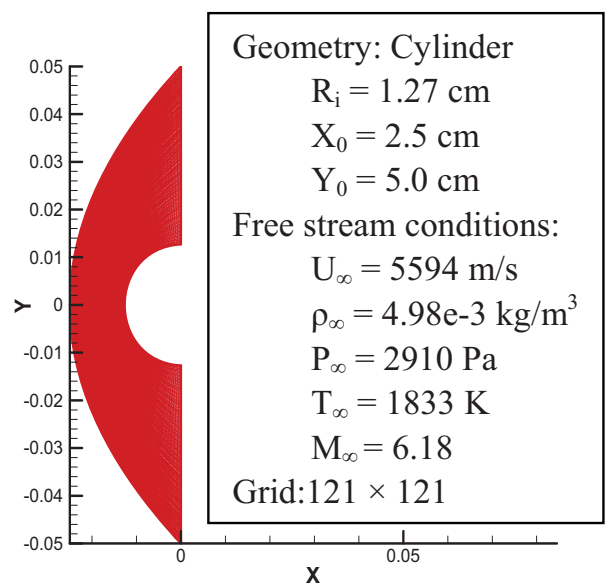

Fig. 29. Geometry and free stream flow conditions.

Our numerical simulation results are compared with the experimental measurement of Hornung obtained from his paper. As shown in Figs. 30 and 31, the shock standoff distance agrees well with experiment and the fringe pattern matches quite well with Hornung's experimental measurements. The test result on this case validated that the implementations of nonquilibrium and reactive flow solver to the high-order shock-fitting code is accurate.

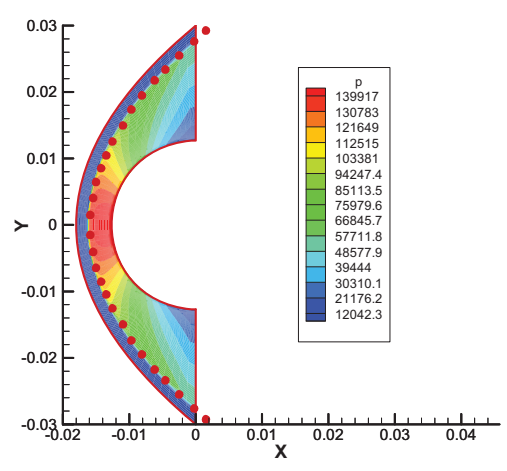

(a)

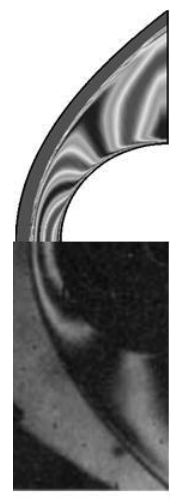

(b)

Fig. 30. Comparisons with experimental measurements: (a) shock standoff distance (Dots: Hornung's measurement); (b) fringe patterns (Lower half: Hornung's experimental measurement). 


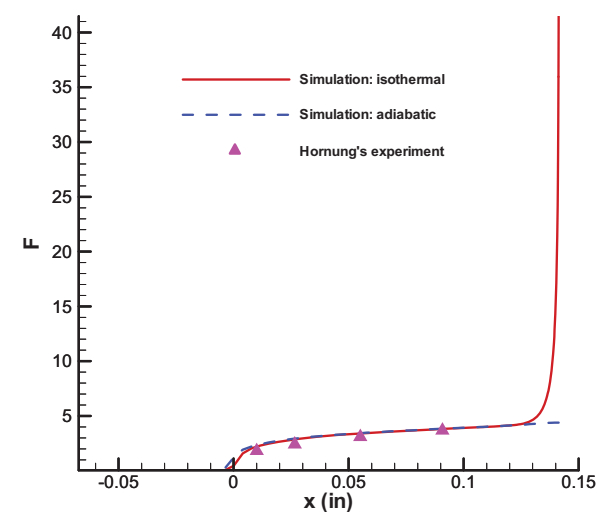

Fig. 31. Quantitative comparison of fringe number along the stagnation line.

\section{CONCLUSIONS AND FUTURE WORK}

During the period of the research project, we have developed and implemented the shock-fitting method for isotropic shock and turbulence interaction problems. Implemented code was optimized and shown to scale well for large number of processors and around six million CPU hours were awarded to us at NERSC and Teragrid/XSEDE computer resources.

We have focused on computing interaction of turbulence with very strong shocks. We have considered flows with mean Mach numbers ranging from 2 to 30. The turbulent Mach number, $M_{t}$, has been varied from 0.12 to 0.38 . Reynolds number based on Taylor microscale, $\mathrm{Re}_{\lambda}$, of upto 52.4. Our studies complement the efforts of our collaborators at Stanford University by considering very strong shocks. Such high mean Mach number values were never considered in past for study of shock turbulence interactions and we observed new trends in some quantities such as streamwise Reynolds stress values. From the computations we observed that while our results follow the trend as reported in literature for low to moderate Mach number flows, the trends are reversed for very strong shocks. Similar to the findings reported by our colleagues at Stanford we also observe that vorticity fluctuations return to isotropy behind the shock. Specifically, the peak of streamwise vorticity fluctuations is observed for shock and turbulence interactions with Mach 2.8 shock. For stronger than Mach 2.8 shocks, there is a decrease in streamwise vorticity fluctuations. The amplification of Reynolds stress $\mathrm{R}_{11}$ decreases as mean Mach number is increased till 8.8, which is consistent with findings of linear interaction analysis. This trend, however, reverses as shock strength is increased beyond Mach 8.8. For stronger than Mach 8.8 shocks, Reynolds stress $\mathrm{R}_{11}$ is amplified as mean Mach number keeps increasing. However, increasing Mach number leads to delay in the return to isotropy in the vorticity fluctuations. The Taylor micsrocale behind the shocks were observed to be decreasing for all the cases considered.

We have also implemented to current shock-fitting code the more realistic 11-species model of air with most recent thermo-chemical models of non-equilibrium air flow, including gas-mixture viscosity, heat conductivity, diffusion coefficients, non-equilibrium reaction rates, and equilibrium constants. The non-equilibrium shock-fitting code can run cases with extremely high translational temperature. This large database of DNS results will be used for developing models and correlations for shock turbulence interactions. 


\section{PUBLICATIONS}

The following publications are completed from work supported by this grant:

X. Wang, P. S. Rawat, and X. Zhong, "Strong shock and turbulence interactions w/ or w/o thermochemical non-equilibrium effects," 65th Annual Meeting of the APS Division of Fluid Dynamics, 2012.

X. Wang and X. Zhong, "A High-Order Shock-Fitting Non-Equilibrium Flow Solver for DNS of Strong Shock and Turbulence Interactions," Seventh International Conference on Computational Fluid Dynamics, Paper ICCFD7-2305, Big Island, Hawaii, July 9-13, 2012.

$\mathrm{X}$. Wang and X. Zhong, "DNS of strong shock and turbulence interactions with thermochemical nonequilibrium effects," AIAA Paper 2012-3162, 2012.

A. Prakash and X. Zhong, "Numerical Simulation of Receptivity of Freestream Disturbances to Hypersonic Boundary Layers with Thermochemical Nonequilibrium", AIAA paper 2012-1085, Jan. 2012.

X. Wang and X. Zhong, "Effect of compressibility on strong shock and turbulence interactions," AIAA Paper 2012-1243, 2012.

A. Prakash, N. Parsons, X. Wang, and X. Zhong, "High-order shock-fitting methods for direct numerical simulation of hypersonic flow with chemical and thermal nonequilibrium," Journal of Computational Physics, Vol. 230, No. 23, pp. 8474-8507, 2011.

$\mathrm{X}$. Wang and X. Zhong, "DNS of strong shock and turbulence interaction including real gas effects," AIAA Paper 2011-3707, 2011.

P. S. Rawat and X. Zhong, "Direct Numerical Simulations of Turbulent Flow Interactions with Strong Shocks Using Shock-Fitting Method," AIAA paper 2011-649, Jan. 2011.

$\mathrm{X}$. Wang and X. Zhong, "Development and validation of a high-order shock-fitting non-equilibrium flow solver," AIAA Paper 2011-0365, 2011.

P. S. Rawat and X. Zhong, "On High-Order Shock-Fitting and Front-Tracking Schemes for Numerical Simulation of Shock-Disturbance Interactions," Journal of Computational Physics, 2010. 229(19): p. 6744-6780.

E. Johnsen, J. Larsson, A. Bhagatwala, W. Cabot, P. Moin, B. Olson, P. Rawat, S. Shankar, B. Sjogreen, H. Yee, X. Zhong and S. Lele, "Assessment of high-resolution methods for numerical simulations of compressible turbulence with shock waves," Journal of Computational Physics, vol. 229, no. 4, pp. 1213$1237,2010$.

A. Prakash, N. Parsons, X. Wang, and X. Zhong, "High- Order Shock- Fitting Methods for Hypersonic Flow with Chemical and Thermal Nonequilibrium," AIAA paper 2010-4997. 2010.

P. S. Rawat and X. Zhong, "Numerical Simulation of Shock-Turbulence Interactions using High-Order Shock-Fitting Algorithms," AIAA paper 2010-114. 2010

$\mathrm{X}$. Wang and X. Zhong, "Nonequilibrium and reactive high-speed flow simulations with a fifth-order WENO scheme," AIAA paper 2009-4041. 2009. 
P. S. Rawat and X. Zhong, "High- Order Shock-Fitting and Front-Tracking Methods for Numerical Simulation of Shock- Disturbance Interactions," AIAA paper 2009-1138. 2009.

P. S. Rawat and X. Zhong, "Numerical Simulations of Strong Shock and Disturbance Interactions Using High-Order Shock-Fitting Algorithms," AIAA paper 2008-0746. 2008.

\section{PERSONNEL}

The following personnel conduct research for the grant and are partially supported by the grant:

1. Professor Xiaolin Zhong, principal investigator.

2. Dr. Xiaowen Wang, research associate.

3. Pradeep S. Rawat, Ph.D. candidate.

4. Akshay Prakash, Ph.D. candidate.

\section{REFERENCES}

1. Kovasznay, L.S.G., Turbulence in supersonic flow. Journal of the Aeronautical Sciences, 1953. 20(10): p. 657-682.

2. Moore, F.K., Unsteady oblique interaction of a shock wave with a plane disturbances. NACA TN-2879 (Also as NACA Rep. 1165), 1953.

3. Kerrebrock, J.L., The interaction of flow discontinuities with small disturbances in a compressible fluid. $\mathrm{PhD}$ thesis, California Institute of Technology, 1956.

4. Goldstein, M.E., Turbulence generated by the interaction of entropy fluctuations with nonuniform mean flows. Journal of Fluid Mechanics, 1979. 93: p. 209-224.

5. Lee, L., Moin, P., and Lele, S. K., Interaction of isotropic turbulence with a shock wave. Report TF-52. Dept. Mech. Eng., Stanford Univ., CA, 1992.

6. Lee, L., Lele, S. K., and Moin, P., Direct numerical simulation of isotropic turbulence interacting with a weak shock wave. Journal of Fluid Mechanics, 1993. 251: p. 533-562.

7. Mahesh, K.A., Lee, L., Lele, S. K., and Moin, P., The interaction of an isotropic field of acoustic waves with a shock wave. Journal of Fluid Mechanics, 1995. 300: p. 383-407.

8. Mahesh, K.A., Lele, S. K., and Moin, P., The influence of entropy fluctuations on the interaction of turbulence with a shock wave. Journal of Fluid Mechanics, 1997. 334: p. 353-379.

9. $\quad$ Fabre, D., Jacquin, L., and Sesterhenn, J., Linear interaction of a cylindrical entropy spot with a shock. Journal of Physics of Fluids A, 2001. 13(8): p. 2403-2422.

10. Pao, S.P., and Salas, M. D., A numerical study of two-dimensional shock vortex interaction. AIAA Paper 81-1205, 1981.

11. Zang, T.A., Hussaini, M. Y., and Bushnell, D. M. , Numerical Computations of Turbulence Amplification in Shock-Wave Interactions. AIAA Journal, 1984. 22(1): p. 13-21.

12. Hussaini, M.Y., Kopriva, D., Salas, M. D., and Zang, T. A., Spectral methods for the Euler equations. II - Chebyshev methods and shock fitting. AIAA Journal, 1987. 23: p. 234-240.

13. Meadows, K.R., Kumar, A., and Hussaini, M. Y., Computational Study on the Interaction between a vortex and a shock Wave. AIAA Journal, 1991. 29(2): p. 174-179.

14. Meadows, K.R., and Casper, J., Computing unsteady shock waves for aeroacoustic applications. AIAA Paper 1993-4329, 1993.

15. Grasso, F., and Pirozzoli, S., Shock-wave-vortex interactions: shock and vortex deformations, and sound production. Theoretical and Computational Fluid Dynamics, 1993. 13(6): p. 421-456. 
16. Andreopoulos, Y., Agui, J. H., and Briassulis, G., Shock wave-turbulence interactions. Annual Review of Fluid Mechanics 2000. 32: p. 309-345.

17. Hannappel, R., and Friedrich, R., Direct numerical simulation of a Mach 2 shock interacting with isotropic turbulence. Applied Scientific Research, 1995. 54: p. 205-221.

18. Jamme, S., Cazalbou, J. B., Torres, F., and Chassaing, P., Direct numerical simulation of the interaction between a shock wave and various types of isotropic turbulence. Flow, Turbulence and Combustion, 2202. 68: p. 227-268.

19. Adams, N.A., and Shariff, K., A high-resolution hybrid compact-ENO scheme for shock turbulence interaction problems. Journal of Computational Physics, 1996. 127(27): p. 57-.

20. Adams, N.A., and Shariff, K., Direct numerical simulation of turbulent compression corner flow. Theoretical and Computational Fluid Dynamics, 1998. 12: p. 109-129.

21. Pirozzoli, S., Conservative hybrid compact-WENO schemes for shock-turbulence interaction. Journal of Computational Physics, 2002. 178(1): p. 81-117.

22. Ducros, F., Ferrand, V., Nicoud, F.,Weber, C., Darracq, D., Gacherieu C., and Poinsot, T., Large eddy simulation of the shock/turbulence interaction. Journal of Computational Physics, 1999. 152: p. 517-549.

23. Lee, S., Lele, S. K., and Moin, P., Interaction of isotropic turbulence with shock waves: effect of shock strength. Journal of Fluid Mechanics, 1997. 340: p. 225-247.

24. Jamme, S., Cazalbou, J. B., Torres, F., and Chassaing, P., Direct numerical simulation of the interaction between a shock wave and various types of isotropic turbulence. Flow, Turbulence and Combustion, 2002. 68: p. 227-268.

25. Lee, T.K., and Zhong, X., Spurious numerical oscillations in simulation of supersonic flows using shock-capturing schemes. AIAA Journal, 1999. 37(3): p. 313-319.

26. Agui, J.H., Shock wave interactions with turbulence and vortices. 1998, PhD Thesis, City University of New York: New York.

27. Lele, S.K., and Larsson, J., Shock-turbulence interaction: What we know and what we can learn from peta-scale simulations. Journal of Physics: Conference Series, 2009. 180(012032).

28. Vincenti, W.G., and Kruger Jr., C. H., Introduction to Physical Gas Dynamics 1967: Krieger Publishing Co, INC.

29. Carpenter, M.H., and Casper, J. H., Accuracy of shock capturing in two spatial dimensions. AIAA Journal, 1999. 37(9): p. 1072-1079.

30. Rawat, P.S., and Zhong, X. Numerical Simulation of Shock-Turbulence Interactions using HighOrder Shock-Fitting Algorithms. in AIAA paper 2010-114. 2010.

31. Rawat, P.S., and Zhong, X. Numerical Simulations of Strong Shock and Disturbance Interactions Using High-Order Shock-Fitting Algorithms. in AIAA paper 2008-0746. 2008.

32. Rawat, P.S., and Zhong, X. High- Order Shock-Fitting and Front-Tracking Methods for Numerical Simulation of Shock- Disturbance Interactions. in AIAA paper 2009-1138. 2009.

33. Rawat, P.S., and Zhong, X., On high-order shock-fitting and front-tracking schemes for numerical simulation of shock-disturbance interactions. Journal of Computational Physics, 2010. 229(19): p. 6744-6780

34. Gupta, R.N., Yos, J. M., Thompson, R. A., and Lee, K-P., A Review of Reaction Rates and Thermodynamic and Transport Properties for an 11-Species Air Model for Chemical and Thermal Nonequilibrium Calculations to 30000 K. 1990, NASA Reference Publication 1232.

35. Yos, J.M., Transport properties of Nitrogen, Hydrogen, Oxygen, and Air to 30000 K. 1963, US Air Force Technical Momorandum RAD-TM-63-7.

36. Park, C., Nonequilibrium hypersonic aerothermodynamics. 1990, New York: Wiley. .

37. Park, C., Jaffe, R. L., and Partridge, H., Chemical-Kinetic Parameters of Hyperbolic Earth Entry. Journal of thermophysics and heat transfer, 2001. 15(1): p. 76-90.

38. McBride, B.J., Zehe, M. J., and Gordon, S., NASA Glenn Coefficients for Calculating Thermodynamic Properties of Individual Species. 2002, NASA TP 2002-211556. 
39. Lee, L., Lele, S. K., and Moin, P., Simulation of spatially evolving compressible turbulence and the applicability of Taylor's Hypothesis. Physics of Fluids A, 1992. 4: p. 1521-1530.

40. Erlebacher, G., Hussaini, Y., Kreiss, H. O., and Sarkar, S., The Analysis and Simulation of Compressible Turbulence. Theoretical and Computational Fluid Dynamics, 1990. 2: p. 73-95.

41. Zhong, X., High-order finite-difference schemes for numerical simulation of hypersonic boundary-layer transition. Journal of Computational Physics, 1998. 144: p. 662-709.

42. Samtaney, R., Pullin, D. I., and Kosovic, B., Direct numerical simulation of decaying compressible turbulence and shocklet statistics. Physics of Fluids, 2001. 13(5): p. 1415-1430.

43. Ristorcelli, J.R., and Blaisedell, G. A., Consistent initial conditions for the DNS of compressible turbulence. Physics of Fluids, 1997. 9(1): p. 4-6.

44. Moin, P., and Mahesh, K., Direct Numerical Simulation: A tool in turbulence research. Annual Review of Fluid Mechanics, 1998. 30: p. 539-578.

45. Larsson, J., and Lele, S.K., Direct numerical simulation of canonical shock/turbulence interaction. Physics of Fluids, 2009. 21(12): p. 126101-126101-12.

46. Poinsot, T.J., and Lele, S. K., Boundary conditions for direct simulations of compressible viscous flow. Journal of Computational Physics, 1992. 101(1): p. 104-129.

47. Lele, S.K., Shock-jump relations in a turbulent flow. Physics of Fluids, 1992. 4: p. 2900-2905.

48. Ribner, H.S., Convection of a pattern of vorticity through a shock wave. NACA TN-2864 (Also as NACA Report 1164 ), 1953.

49. Ribner, H.S., Shock-turbulence interaction and the generation of noise. NACA TN-3255 (Also as NACA Report 1233), 1954.

50. Andreopoulos, Y., Agui, J. H., and Briassulis, G., Shock wave-turbulence interactions. Annual Review of Fluid Mechanics, 2000. 32: p. 309-345.

51. Hash, D., Olejniczak, J, Wright, M. J., Dinish, P., Pulsonetti, M., Hollis, B. R., Gnoffo, P. A., Barnhard, M., Nompelis, I., and Candler, G., FIRE II Calculations for Hypersonic Nonequilibrium Aerothermodynamics Code Validation: DPLR, LAURA, and US3D. 2007, AIAA 2007-0605.

52. Gnoffo, P.A., Gupta, R. N., and Shinn, J. L., Conservation equations and physical models for hypersonic air flows in thermal and chemical nonequilibrium. 1989, NASA Technical Paper 2867.

53. Hudson, M.L., Linear Stability of Hypersonic Flows in Thermal and Chemical Nonequilibrium. 1996, North Carolina State University.

54. Hornung, H.G., Non-equilibrium dissociating nitrogen flow over spheres and circular cylinders. Journal of Fluid Mechanics, 1972. 53(1): p. 149-176. 